\title{
The sutured Floer polytope and taut depth-one foliations
}

\author{
IRIDA ALTMAN
}

For closed 3-manifolds, Heegaard Floer homology is related to the Thurston norm through results due to Ozsváth and Szabó, Ni, and Hedden. For example, given a closed 3-manifold $Y$, there is a bijection between vertices of the $\mathrm{HF}^{+}(Y)$ polytope carrying the group $\mathbb{Z}$ and the faces of the Thurston norm unit ball that correspond to fibrations of $Y$ over the unit circle. Moreover, the Thurston norm unit ball of $Y$ is dual to the polytope of $\widehat{H F(Y)}$.

We prove a similar bijection and duality result for a class of 3-manifolds with boundary called sutured manifolds. A sutured manifold is essentially a cobordism between two possibly disconnected surfaces with boundary $R_{+}$and $R_{-}$. We show that there is a bijection between vertices of the sutured Floer polytope carrying the group $\mathbb{Z}$ and equivalence classes of taut depth-one foliations that form the foliation cones of Cantwell and Conlon. Moreover, we show that a function defined by Juhász, which we call the geometric sutured function, is analogous to the Thurston norm in this context. In some cases, this function is an asymmetric norm and our duality result is that appropriate faces of this norm's unit ball subtend the foliation cones.

An important step in our work is the following fact: a sutured manifold admits a fibration or a taut depth-one foliation whose sole compact leaves are exactly the connected components of $R_{+}$and $R_{-}$if and only if there is a surface decomposition of the sutured manifold resulting in a product manifold.

57M27; 57R30, 57R58

\section{Introduction}

\subsection{Overview}

The inspiration for this paper comes from a connection between two invariants of closed 3-manifolds: the Thurston norm (see Thurston [28]) and Heegaard Floer homology (see Ozsváth and Szabó [23]). Thurston defined the norm in the 1970s using purely topological terms. Twenty years later, Ozsváth and Szabó developed the Heegaard Floer homology machinery, which draws its roots from complicated pseudoholomorphic 
techniques. In this paper, we aim to show that there is a similar connection between two invariants of a type of 3-manifolds with boundary called sutured manifolds.

Let us first consider the closed case. Let $Y$ be a closed oriented 3-manifold. Recall that the Thurston norm is a seminorm on the homology group $H_{2}(Y ; \mathbb{R})$ that measures the minimal "complexity" of surfaces representing an integral homology class. Rational rays through certain faces of the Thurston norm unit ball, called fibred faces, correspond to fibrations of the 3-manifold over the unit circle (if there are any). The Heegaard Floer homology invariant $\mathrm{HF}^{+}(Y)$ is a bigraded abelian group, with one of the gradings given by a set of so called $\operatorname{Spin}^{c}$ structures of $Y$ which can be identified with $H^{2}(Y ; \mathbb{Z})$. The support of $H F^{+}(Y)$ is the set of elements $\mathfrak{s} \in H^{2}(Y ; \mathbb{Z})$ for which $H F^{+}(Y, \mathfrak{s})$ is nonzero. The convex hull of the support of $\mathrm{HF}^{+}(Y)$ is a polytope in $H^{2}(Y ; \mathbb{R})$.

The connection between the two mentioned invariants is the following: the fibred faces of the Thurston norm ball correspond bijectively to vertices $\mathfrak{s}$ of the $H F^{+}$polytope that support $H F^{+}(Y, \mathfrak{s})=\mathbb{Z}$ (see Ni [21, Theorem 1.1]). ${ }^{1}$ This result generalised a theorem of Ghiggini [15, Theorem 1.4] that a genus-one knot $K$ in $S^{3}$ is fibred if and only if another appropriate Heegaard Floer group $\widehat{H F K}(K, 1)$ is isomorphic to $\mathbb{Z}$. In summary, we have the following correspondences:

(1) $\quad Y$ fibres over $S^{1} \stackrel{\text { Thurston }}{\Longleftrightarrow}$ fibred face $\stackrel{\mathrm{Ni}}{\Longleftrightarrow}$ vertex $\mathfrak{s}$ with $H F^{+}(Y, \mathfrak{s})=\mathbb{Z}$

Let us now consider the case of sutured manifolds. A sutured manifold $(M, \gamma)$ is a cobordism $M$ between two possibly disconnected surfaces with boundary $R_{+}(\gamma)$ and $R_{-}(\gamma)$, and a set $\gamma$ of pairwise disjoint annuli and tori on $\partial M$ such that $\gamma \cup$ $R_{+}(\gamma) \cup R_{-}(\gamma)=\partial M$. (The surfaces and 3-manifold also have to satisfy certain orientability conditions.) We show that there is a correspondence between taut, depthone foliations of a sutured manifold and the polytope of sutured Floer homology developed by Juhász [17]. Gabai defined and developed taut, finite depth foliations for sutured manifolds in the 1980s. Twenty years later, Juhász [17] extended the "hat flavour" of Heegaard Floer homology for closed 3-manifolds to (balanced) sutured manifolds. But before we can give a statement analogous to (1) we introduce the key ingredients.

We call $\mathcal{F}$ a foliation of $(M, \gamma)$ if the leaves of $\mathcal{F}$ are transverse to $\gamma$ and tangential to $R(\gamma):=\partial M \backslash \operatorname{Int}(\gamma)$. For example, if $M$ is a solid torus and $\gamma=\partial M$, then the fibration of $M=S^{1} \times D^{2} \rightarrow S^{1}$ given by the projection onto the first coordinate is a depth-zero foliation of $(M, \gamma)$. We only work with foliations that are smooth and transversely oriented.

\footnotetext{
${ }^{1}$ Note that the Thurston (semi)norm of a homology class represented by a torus is zero. Hence if a manifold fibres with fibre a torus, there is no fibred face of the Thurston norm unit ball corresponding to this fibration. Ni's proof works under the assumption that the fibre has genus greater than one.
} 
Cantwell and Conlon showed [10] that taut depth-one foliations form open, convex, polyhedral cones in $H^{1}(M ; \mathbb{R})=H_{2}(M, \partial M ; \mathbb{R})$ called foliation cones. Note that rational rays through the fibred faces of the Thurston norm unit ball also live in open, convex, polyhedral cones (which we could call fibred cones) that are subtended by the fibred faces. So similarly to the first correspondence in (1), we have

$$
(M, \gamma) \text { has taut depth-1 foliation } \stackrel{\text { Cantwell-Conlon }}{\Longleftrightarrow} \text { foliated face. }
$$

On the other hand, sutured Floer homology associates to a balanced sutured manifold $(M, \gamma)$ a finitely generated abelian group denoted by $\operatorname{SFH}(M, \gamma)$. One of the gradings is given by a set of so called relative $\operatorname{Spin}^{c}$ structures of $(M, \gamma)$, which can be identified with $H^{2}(M, \partial M ; \mathbb{Z})$. Then the support of $\operatorname{SFH}(M, \gamma)$ is defined to be the set of elements $\mathfrak{s} \in H^{2}(M, \partial M ; \mathbb{Z})$ for which $\operatorname{SFH}(M, \gamma, \mathfrak{s})$ is nonzero. The convex hull of the support is the sutured Floer polytope $P(M, \gamma)$ living in $H^{2}(M, \partial M ; \mathbb{R})$ [19].

Our connection between the two theories, given in Theorem A, is that the foliation cones of $(M, \gamma)$ correspond bijectively to vertices $\mathfrak{s}$ of $P(M, \gamma)$ that support $\operatorname{SFH}(M, \gamma, \mathfrak{s})=\mathbb{Z}$. Thus, with Theorem A, we have the following set of correspondences in (1):

$$
\begin{aligned}
(M, \gamma) \text { has taut depth-1 foliation } \stackrel{\text { Cantwell-Conlon }}{\stackrel{\text { Theorem A }}{\Longleftrightarrow} \text { foliated face }} \text { vertex } \mathfrak{s} \text { with } \operatorname{SFH}(M, \gamma, \mathfrak{s})=\mathbb{Z}
\end{aligned}
$$

The relationships between the old and new theories do not stop there. In particular, Ozsváth and Szabó [22, Theorem 1.1] showed that the Thurston norm unit ball is dual to the polytope of $\underline{\widehat{H F}}(Y)$, another flavour of Heegaard Floer homology with so-called twisted coefficients. In Theorem B, we show that the foliation cones of Cantwell and Conlon are subtended by faces of the dual sutured Floer polytope. Note that the foliation cones do not form a polytope, and this agrees with the fact that the sutured Floer polytope is defined up to translation in $H^{2}(M, \partial M ; \mathbb{R})$. In the closed case there is no such ambiguity, as both the Thurston norm and $\underline{H F}(Y)$ are well-defined, that is, "centred" around the origin of their ambient vector space.

It may be helpful to bear in mind the following dictionary of terms between the closed case and the case of balanced sutured manifolds, especially if one is already familiar with the former.

\begin{tabular}{c|c}
$Y$ closed 3-manifold & $(M, \gamma)$ balanced sutured manifold \\
\hline Thurston norm $x$ & geometric sutured function $y_{t}$ \\
$Y$ fibres over $S^{1}$ & $(M, \gamma)$ admits a taut depth-one foliation \\
fibred face of $x$ unit norm ball & foliated face of $y_{t}$ "unit norm ball" \\
vertex $\mathfrak{s}$ with $H F^{+}(Y, \mathfrak{s})=\mathbb{Z}$ & vertex $\mathfrak{s}$ with $S F H(M, \gamma, \mathfrak{s})=\mathbb{Z}$ \\
$\underline{\hat{H F}}(Y)$ polytope & $\operatorname{SFH}(M, \gamma)$ polytope
\end{tabular}


We have not yet talked about the geometric sutured function $y_{t}$, which is a map $H_{2}(M, \partial M ; \mathbb{R}) \rightarrow \mathbb{R}$, but it is easiest if we delay its introduction until Section 2.5. The function $y_{t}$ can take negative values, hence it may not have a unit norm ball per se. However, when $y_{t}$ is a seminorm, then its unit ball is dual to the sutured polytope $P(M, \gamma)$; see Corollary 2.16 for details.

\subsection{Statements of results}

The polytope $P(M, \gamma)$ is said to have an extremal $\mathbb{Z}$ at $\mathfrak{s}$ if $\mathfrak{s}$ is a vertex of the polytope and $\operatorname{SFH}(M, \gamma, \mathfrak{s})=\mathbb{Z}$. Saying that $\mathfrak{s}$ is extremal with respect to a homology class $\alpha \in H_{2}(M, \partial M ; \mathbb{R})$ means that $\alpha(\mathfrak{s})>\alpha(\mathfrak{t})$ for any other vertex $\mathfrak{t}$ of the polytope. Set $R(\gamma):=R_{+}(\gamma) \cup R_{-}(\gamma)$ and $M_{0}:=M \backslash R(\gamma)$. If $\mathcal{F}$ is a depth-one foliation, then the manifold $M_{0}$ fibres over $S^{1}$ and this fibration defines an element $\lambda(\mathcal{F}) \in H^{1}(M ; \mathbb{R})$ (Lemma 3.5). Moreover, $\lambda(\mathcal{F})$ gives rise to a "foliation ray of $\mathcal{F}$ " in $H^{1}(M ; \mathbb{R})=$ $H_{2}(M, \partial M ; \mathbb{R})$ given by $r \cdot(P D \circ \lambda(\mathcal{F}))$ for $r \in \mathbb{R}^{\geq 0}$, and this foliation ray is contained in a foliation cone of Cantwell and Conlon. Here we denote by $P D$ the PoincaréLefschetz duality map.

We can now state the first of our two main theorems.

Theorem A Suppose $(M, \gamma)$ is a taut, strongly balanced sutured manifold with $H_{2}(M ; \mathbb{Z})=0$, and let $P(M, \gamma)$ denote its sutured polytope. Then $P(M, \gamma)$ has an extremal $\mathbb{Z}$ at a $\operatorname{Spin}^{c}$ structure $\mathfrak{s}$ if and only if there exists a taut depth-one foliation $\mathcal{F}$ of $(M, \gamma)$ whose sole compact leaves are the connected components of $R(\gamma)$ and such that $\mathfrak{s}$ is extremal with respect to $P D \circ \lambda(\mathcal{F})$.

The condition that $H_{2}(M ; \mathbb{Z})=0$ is purely technical; it carries over from theorems about how sutured Floer homology behaves under surface decompositions. For a more detailed discussion see Remark 4.13.

As we mentioned above, the Thurston norm unit ball is dual to the polytope of $\underline{\widehat{H F}}(Y)$. Since the Thurston norm is centrally symmetric, this duality result recovers the symmetries of $\widehat{H F}$. However, the sutured Floer polytope is not symmetric in general; for examples see Section 4.3 below and Friedl, Juhász and Rasmussen [12, Section 8]. So there is no hope that $P(M, \gamma)$ is the dual of a (semi)norm unit ball. Moreover, there is no canonical identification of $\operatorname{Spin}^{c}(M, \gamma)$ with $H^{2}(M, \partial M ; \mathbb{Z})$, so $P(M, \gamma)$ is defined in $H^{2}(M, \partial M ; \mathbb{R})$ only up to translation.

Nevertheless, under certain conditions (for example when $H_{2}(M ; \mathbb{Z})=0$ ) we can define a geometric sutured function $y_{t}$ that is analogous to the Thurston norm. This function $y_{t}$ is a sum of two symmetric terms and an asymmetric term that reflects 
the choice of identification $\operatorname{Spin}^{c}(M, \gamma) \rightarrow H^{2}(M, \partial M ; \mathbb{Z}$ ) (see Section 2.5). For those already familiar with Juhász's work, $y_{t}$ is defined using the function $c(S, t)$ that depends on the topology of a properly embedded surface $S$ in $M$, and on the trivialisation $t$ of a particular plane bundle (see Juhász [19, Definition 3.16] or (6) below). However, we advertise the geometric nature of $P(M, \gamma)$ explicitly by putting it side by side with other seminorms for 3-manifolds.

The preceding discussion implies that there is no obvious definition of a dual sutured polytope. Even so, we can always obtain the dual sutured cones $Q(M, \gamma)$ of the polytope. In general, we can define the dual cones $Q$ of any polytope $P$. Let $P$ be given, up to translation, by vertices $v_{1}, \ldots, v_{n}$ living in a vector space $V$ over some field $\mathbb{F}$. The set $Q$ is a collection of polyhedral cones $Q_{1}, \ldots, Q_{n}$ in the dual space $V^{*}=\operatorname{Hom}(V, \mathbb{F})$, where

$$
Q_{i}:=\left\{v^{*} \in V^{*}: v^{*}\left(v_{i}-v_{j}\right)>0 \text { for } i \neq j\right\} .
$$

In particular, the cones of $Q(M, \gamma)$ correspond to vertices of $P(M, \gamma)$. The cones that correspond to extremal $\mathbb{Z}$ vertices of $P(M, \gamma)$ are referred to as the extremal $\mathbb{Z}$ cones and denoted by $Q_{\mathbb{Z}}(M, \gamma)$.

Finally, we state the second main theorem.

Theorem B Let $(M, \gamma)$ be a taut, strongly balanced sutured manifold with $H_{2}(M)=0$. The extremal $\mathbb{Z}$ cones $Q_{\mathbb{Z}}(M, \gamma)$ are precisely the foliation cones defined by Cantwell and Conlon in [10].

Most interesting sutured manifolds are strongly balanced, so this is not a significant restriction in Theorem B. For a precise definition of strongly balanced, see Definition 2.3.

Theorem B provides a connection between two areas of low-dimensional topology that use seemingly different tools. The construction of sutured Floer homology relies on pseudoholomorphic techniques which form the basis of Heegaard Floer homology, whereas Cantwell and Conlon prove the existence of foliation cones by using foliation currents (see Schwartzmann [26] and Sullivan [27]).

Lastly, we would like to mention a key step in our work stated in Lemma C. In the case of a closed 3-manifold $Y$ that fibres over $S^{1}$, cutting $Y$ along a fibre yields a product manifold homeomorphic to fibre $\times I$. Conversely, given an automorphism of a closed surface $\varphi: S \rightarrow S$, the mapping torus fibres over $S^{1}$ with fibre $S$. In the case of a sutured manifold $(M, \gamma)$, Lemma $\mathrm{C}$ states that cutting $(M, \gamma)$ along a properly embedded surface $S$ gives a product manifold if and only if $S$ is either a fibre of a fibration $M \rightarrow S^{1}$ or can be made into a leaf of a depth-one foliation of $(M, \gamma)$ by 
an operation called "spinning". We give the precise definitions of all the terminology used in Lemma $\mathrm{C}$ at appropriate places throughout Sections 2 and 3.

Lemma C Suppose $(M, \gamma)$ is a connected sutured manifold. Let $(M, \gamma) \stackrel{S}{\rightsquigarrow}\left(M^{\prime}, \gamma^{\prime}\right)$ be a surface decomposition along $S$ such that $\left(M^{\prime}, \gamma^{\prime}\right)$ is taut. Then $\left(M^{\prime}, \gamma^{\prime}\right)$ is a product sutured manifold if and only if either

(i) $R(\gamma)=\varnothing$ and $S$ is the fibre of a depth-zero foliation $\mathcal{F}$ given by a fibration $\pi: M \rightarrow S^{1}$, or

(ii) $R(\gamma) \neq \varnothing$ and $S$ can be spun along $R(\gamma)$ to be a leaf of a depth-one foliation $\mathcal{F}$ of $(M, \gamma)$ whose sole compact leaves are the connected components of $R(\gamma)$.

Up to equivalence, all depth-zero foliations of $(M, \gamma)$, and all depth-one foliations of $(M, \gamma)$ whose sole compact leaves are the connected components of $R(\gamma)$, are obtained from a surface decomposition resulting in a product sutured manifold.

Remark 1.1 The gist of Lemma $\mathrm{C}$ is known to experts (private correspondence with Conlon and Gabai), but the author was unable to find any written references. Cantwell and Conlon are preparing a paper [7] exploring the relationship of sutured manifold decomposition and foliations from the perspective of staircases and junctures that will include a proof of Lemma C. Below we give our own proof of Lemma C, together with the minimal necessary background from foliation theory.

\subsection{Organisation of the article}

The article is organised as follows. In Section 2, we give the background on the sutured Floer polytope and surface decompositions, followed by a survey of seminorms on the homology of 3-manifolds. In Section 3, we describe Gabai's construction of depth-one foliations, followed by the theory of junctures and spiral staircases of foliations, and an introduction to Cantwell and Conlon's foliation cones. Finally, in Section 4 we prove Lemma C, Theorems A and B (in that order), and we present a few examples that illustrate the duality between foliation cones and the sutured Floer polytope. Notational conventions are explained at the beginning of Section 2.

\subsection{Acknowledgements}

I would like to thank my $\mathrm{PhD}$ advisers Stefan Friedl and András Juhász for suggesting this project to me, as well as for many helpful discussions and suggestions. Many thanks to Lawrence Conlon for explaining aspects of depth-one foliation theory, in particular, 
the concepts of spiral staircase and junctures, and for his insightful correspondence on foliation cones. I thank my $\mathrm{PhD}$ adviser Saul Schleimer for his interest in my work.

I owe much gratitude to the University of Warwick and the Warwick Mathematics Institute for generously supporting me through a Warwick Postgraduate Research Scholarship, as well as to the Department for Pure Mathematics and Mathematical Statistics in Cambridge for their hospitality.

\section{Sutured manifolds}

This section gives the background to understanding the sutured Floer homology side of the duality statement. We begin with the basic definitions of sutured manifolds and the operation of surface decomposition (see Gabai [13]). This is followed by a brief review of sutured Floer homology and relative $\operatorname{Spin}^{c}$ structures (see Juhász [17]), as well as the definition of the sutured Floer polytope (see Juhász [19]). Then there is a description of how sutured Floer homology behaves under decompositions along "well-behaved" surfaces (see Juhász [18]). The section ends with a survey of seminorms for 3-manifolds: the Thurston norm (see Thurston [28]), the generalised Thurston norm (see Scharlemann [25]), the sutured Thurston norm (see Cantwell and Conlon [8]), the sutured seminorm (see Juhász [19]), and finally what we call the geometric sutured function.

\subsection{Notation}

If two topological spaces $W$ and $X$ are homeomorphic, we write $W \cong X$. If $U$ is an open set in $X$, then $\bar{U}$ denotes the closure of $U$ in the topology of $X$. Denote by $|X|$ the number of connected components of $X$.

All homology groups are assumed to be given with $\mathbb{Z}$-coefficients unless otherwise stated. Let $M$ be an $n$-manifold with boundary. Then $P D$ denotes the PoincaréLefschetz duality map $H_{*}(M, \partial M) \rightarrow H^{n-*}(M)$. As this map is an isomorphism, we simplify the notation and also call the inverse map $P D$; it is obvious from the context to which map we are referring.

Let $M$ be an $n$-manifold, and $L \subset M$ a codimension- 1 submanifold. Then a tubular neighbourhood of $L$, denoted by $N(L)$, is often parametrized as $L \times(0,1)$. We write $L \times s$ to mean $L \times\{s\}:=\{(x, s) \in M: x \in L\}$ for some $s \in[0,1]$. Similarly, in general, when $J \times(a, b) \times(c, d)$ is the parametrisation of a tubular neighbourhood of a codimension-2 submanifold $J \subset M$, we write $J \times s \times t$ or $(J, s, t)$ to mean the codimension-2 manifold $J \times\{s\} \times\{t\}$, where $(s, t) \in[a, b] \times[c, d]$. 


\subsection{Sutured manifolds}

Sutured manifolds were defined by Gabai [13, Definition 2.6].

Definition 2.1 A sutured manifold $(M, \gamma)$ is a compact oriented 3-manifold $M$ with boundary together with a set $\gamma \subset \partial M$ of pairwise disjoint annuli $A(\gamma)$ and tori $T(\gamma)$. Furthermore, in the interior of each component of $A(\gamma)$ one fixes a suture, that is, a homologically nontrivial oriented simple closed curve. We denote the union of the sutures by $s(\gamma)$.

Finally, every component of $R(\gamma):=\partial M \backslash \operatorname{Int}(\gamma)$ is oriented. Define $R_{+}(\gamma)$ (or $\left.R_{-}(\gamma)\right)$ to be those components of $\partial M \backslash \operatorname{Int}(\gamma)$ whose normal vectors point out of (into) $M$. The orientation on $R(\gamma)$ must be coherent with respect to $s(\gamma)$, that is, if $\delta$ is a component of $\partial R(\gamma)$ and is given the boundary orientation, then $\delta$ must represent the same homology class in $H_{1}(\gamma)$ as some suture.

Sutured Floer homology is defined on a wide subclass of sutured manifolds called balanced sutured manifolds, whereas the sutured Floer polytope is defined for strongly balanced sutured manifolds.

Definition 2.2 (Juhász [17, Definition 2.2]) A balanced sutured manifold is a sutured manifold $(M, \gamma)$ such that $M$ has no closed components, the equality $\chi\left(R_{+}(\gamma)\right)=$ $\chi\left(R_{-}(\gamma)\right)$ holds, and the map $\pi_{0}(A(\gamma)) \rightarrow \pi_{0}(\partial M)$ is surjective.

Definition 2.3 (Juhász [18, Definition 3.5]) A strongly balanced sutured manifold is a balanced sutured manifold $(M, \gamma)$ such that for every component $F$ of $\partial M$ the equality $\chi\left(F \cap R_{+}(\gamma)\right)=\chi\left(F \cap R_{-}(\gamma)\right)$ holds.

The most frequently studied and most "interesting" examples of sutured manifolds are all strongly balanced. A trivial example is the product sutured manifold given by $(\Sigma \times I, \partial \Sigma \times I)$, where $\Sigma$ is a surface with boundary and with no closed components.

Other simple examples are obtained from any closed, connected 3-manifold by removing a finite number of 3-balls and adding one trivial suture to each spherical boundary component. Less trivial examples are those of link complements in closed 3-manifolds with sutures consisting of an even number of $(p, q)$-curves on the toroidal components, as well as the complements of surfaces in closed 3-manifolds, endowed with sutures derived from the boundary of the surface (for example, the complement of a Seifert surface of a knot). ${ }^{2}$

\footnotetext{
${ }^{2}$ More precisely, one does not take the complement of surface $\Sigma$ in a closed 3-manifold $Y$, but instead the complement of a double collar neighbourhood $\Sigma \times(-1,1)$ of $\Sigma$. Then the sutures are the curves corresponding to $\partial S \times\{0\}$ on $\partial(Y \backslash \Sigma \times(-1,1))$.
} 
Definition 2.4 A sutured manifold $(M, \gamma)$ is said to be taut if $M$ is irreducible, and $R(\gamma)$ is incompressible and Thurston-norm minimising in $H_{2}(M, \gamma)$.

Lastly, we define the operation of decomposing sutured manifolds into simpler pieces which was introduced by Gabai [13, Definition 3.1].

Definition 2.5 Let $(M, \gamma)$ be a sutured manifold and $S$ a properly embedded surface in $M$ such that for every component $\lambda$ of $S \cap \gamma$ one of (i)-(iii) holds:

(i) $\lambda$ is a properly embedded nonseparating arc in $\gamma$.

(ii) $\lambda$ is simple closed curve in an annular component $A$ of $\gamma$ in the same homology class as $A \cap s(\gamma)$.

(iii) $\lambda$ is a homotopically nontrivial curve in a toroidal component $T$ of $\gamma$, and if $\delta$ is another component of $T \cap S$, then $\lambda$ and $\delta$ represent the same homology class in $H_{1}(T)$.

Then $S$ defines a sutured manifold decomposition

$$
(M, \gamma) \stackrel{S}{\rightsquigarrow}\left(M^{\prime}, \gamma^{\prime}\right),
$$

where $M^{\prime}:=M \backslash \operatorname{Int}(N(S))$ and

$$
\begin{aligned}
\gamma^{\prime} & :=\left(\gamma \cap M^{\prime}\right) \cup N\left(S_{+}^{\prime} \cap R_{-}(\gamma)\right) \cap N\left(S_{-}^{\prime} \cap R_{+}(\gamma)\right), \\
R_{+}\left(\gamma^{\prime}\right) & :=\left(\left(R_{+}(\gamma) \cap M^{\prime}\right) \cup S_{+}^{\prime}\right) \backslash \operatorname{Int}\left(\gamma^{\prime}\right), \\
R_{-}\left(\gamma^{\prime}\right) & :=\left(\left(R_{-}(\gamma) \cap M^{\prime}\right) \cup S_{-}^{\prime}\right) \backslash \operatorname{Int}\left(\gamma^{\prime}\right),
\end{aligned}
$$

where $S_{+}^{\prime}\left(S_{-}^{\prime}\right)$ are the components of $\partial N(S) \cap M^{\prime}$ whose normal vector points out of (into) $M$.

The manifolds $S_{+}$and $S_{-}$are defined in the obvious way as copies of $S$ embedded in $\partial M^{\prime}$ that are obtained by cutting $M$ along $S$.

\subsection{Sutured Floer homology and the sutured Floer polytope}

First, here is some background on $\operatorname{Spin}^{c}$ structures. The following definition of relative $\operatorname{Spin}^{c}$ structures originates from Turaev's work [30], but in the current phrasing comes from Juhász [17]. For proofs and details we refer to Juhász's papers [17; 18; 19].

Fix a Riemannian metric on $(M, \gamma)$. Let $v_{0}$ denote a nonsingular vector field on $\partial M$ that points into $M$ on $R_{-}(\gamma)$ and out of $M$ on $R_{+}(\gamma)$, and that is equal to the 
gradient of a height function $s(\gamma) \times I \rightarrow I$ on $\gamma$. The space of such vector fields is contractible.

A relative $\operatorname{Spin}^{c}$ structure is defined to be a homology class of vector fields $v$ on $M$ such that $v \mid \partial M$ is equal to $v_{0}$. Here two vector fields $v$ and $w$ are said to be homologous if there exists an open ball $B \subset \operatorname{Int}(M)$ such that $v$ and $w$ are homotopic through nonsingular vector fields on $M \backslash B$ relative to the boundary. There is a free and transitive action of $H_{1}(M)=H^{2}(M, \partial M)$ on $\operatorname{Spin}^{c}(M, \gamma)$ given by Reeb tubularization (see Turaev [30, page 639]). This action makes the set $\operatorname{Spin}^{c}(M, \gamma)$ into an $H_{1}(M)$-torsor. From now on, we refer to a map $\iota: \operatorname{Spin}^{c}(M, \gamma) \rightarrow H_{1}(M)$ as an identification of the two sets if $\iota$ is an $H_{1}(M)$-equivariant bijection. Note that $\iota$ is completely defined by which element $\mathfrak{s} \in \operatorname{Spin}^{c}(M, \gamma)$ it sends to $0 \in H_{1}(M)$ (or any other fixed element of $\left.H_{1}(M)\right)$.

The perpendicular two-plane field $v_{0}^{\perp}$ is trivial on $\partial M$ if and only if $(M, \gamma)$ is strongly balanced (see Juhász [18, Proposition 3.4]). Suppose that $(M, \gamma)$ is strongly balanced. Define $T(M, \gamma)$ to be the set of trivialisations of $v_{0}^{\perp}$. Let $t \in T(M, \gamma)$. Then there is a map dependent on the choice of trivialisation,

$$
c_{1}(\cdot, t): \operatorname{Spin}^{c}(M, \gamma) \longrightarrow H^{2}(M, \partial M),
$$

where $c_{1}(\mathfrak{s}, t)$ is defined to be the relative Euler class of the vector bundle $v^{\perp} \rightarrow M$ with respect to a partial section coming from a trivialisation $t$. So $c_{1}(\mathfrak{s}, t)$ is the first obstruction to extending the trivialisation $t$ of $v_{0}^{\perp}$ to a trivialisation of $v^{\perp}$. Here $v$ is a vector field on $M$ representing the homology class $\mathfrak{s}$.

Sutured Floer homology associates to a given balanced sutured manifold $(M, \gamma)$ a finitely generated bigraded abelian group denoted by $\operatorname{SFH}(M, \gamma)$. The group $\operatorname{SFH}(M, \gamma)$ is graded by the relative $\operatorname{Spin}^{c}$ structures $\mathfrak{s} \in \operatorname{Spin}^{c}(M, \gamma)$, and has a relative $\mathbb{Z}_{2}$ grading. In particular, for each $\mathfrak{s} \in \operatorname{Spin}^{c}(M, \gamma)$ there is a well-defined abelian group $\operatorname{SFH}(M, \gamma, \mathfrak{s})$ (see Juhász [17]), and the direct sum of these groups forms the sutured Floer homology of $(M, \gamma)$. That is,

$$
\operatorname{SFH}(M, \gamma):=\bigoplus_{\mathfrak{s} \in \operatorname{Spin}^{c}(M, \gamma)} \operatorname{SFH}(M, \gamma, \mathfrak{s}) .
$$

A very important property of sutured Floer homology is that it detects the product sutured manifold. In the following theorem we put together [17, Proposition 9.4] and [18, Theorem 9.7].

Theorem 2.6 An irreducible balanced sutured manifold $(M, \gamma)$ is a product manifold if and only if $\operatorname{SFH}(M, \gamma)=\mathbb{Z}$. 
Note that the statement would be false without the word irreducible: if $P(1)$ is the Poincaré homology sphere with a 3-ball removed and a single suture along the spherical boundary, then $\operatorname{SFH}(P(1))=\mathbb{Z}$ (see Juhász [17, Remark 9.5]), but $P(1)$ is not a product (and not irreducible by definition).

We now have all the ingredients required to define the sutured Floer polytope. Let $S(M, \gamma)$ be the support of the sutured Floer homology of $(M, \gamma)$. That is,

$$
S(M, \gamma):=\left\{\mathfrak{s} \in \operatorname{Spin}^{c}(M, \gamma): \operatorname{SFH}(M, \gamma, \mathfrak{s}) \neq 0\right\} .
$$

Consider the map $i: H^{2}(M, \partial M ; \mathbb{Z}) \rightarrow H^{2}(M, \partial M ; \mathbb{R})$ induced by the inclusion $\mathbb{Z} \hookrightarrow \mathbb{R}$. For $t$ a trivialisation of $v_{0}^{\perp}$, define

$$
C(M, \gamma, t):=\left\{i \circ c_{1}(\mathfrak{s}, t): \mathfrak{s} \in S(M, \gamma)\right\} \subset H^{2}(M, \partial M ; \mathbb{R}) .
$$

Definition 2.7 The sutured Floer polytope $P(M, \gamma, t)$ with respect to $t$ is defined to be the convex hull of $C(M, \gamma, t)$.

Next, we have that $c_{1}\left(\mathfrak{s}, t_{1}\right)-c_{1}\left(\mathfrak{s}, t_{2}\right)$ is an element of $H^{2}(M, \partial M)$ dependent only on the trivialisations $t_{1}$ and $t_{2}$ (see Juhász [19, Lemma 3.11]), and therefore we may write $P(M, \gamma)$ to mean the polytope in $H^{2}(M, \partial M ; \mathbb{R})$ up to translation.

It is important to note that $c_{1}$ "doubles the distances". Namely, for a fixed trivialisation $t$ and $\mathfrak{s}_{1}, \mathfrak{s}_{2} \in \operatorname{Spin}^{c}(M, \gamma)$, Juhász [19, Lemma 3.13] says that

$$
c_{1}\left(\mathfrak{s}_{1}, t\right)-c_{1}\left(\mathfrak{s}_{2}, t\right)=2\left(\mathfrak{s}_{1}-\mathfrak{s}_{2}\right),
$$

where $\mathfrak{s}_{1}-\mathfrak{s}_{2}$ is the unique element $h \in H^{2}(M, \partial M)$ such that $\mathfrak{s}_{1}=h+\mathfrak{s}_{2}$. Such an element exists and is unique by definition of an $H^{2}(M, \partial M)$-torsor.

Let $t \in T(M, \gamma)$. Then an element $\alpha \in H_{2}(M, \partial M ; \mathbb{R})$ defines subsets $P_{\alpha}(M, \gamma, t)$ and $C_{\alpha}(M, \gamma, t)$ of $P(M, \gamma, t)$ and $C(M, \gamma, t)$, respectively [19, page 17]. First, set

$$
c(\alpha, t):=\min \{\langle c, \alpha\rangle: c \in P(M, \gamma, t)\} .
$$

Then there is a subset $H_{\alpha} \subset H^{2}(M, \partial M ; \mathbb{R})$ given by

$$
H_{\alpha}:=\left\{x \in H^{2}(M, \partial M ; \mathbb{R}):\langle x, \alpha\rangle=c(\alpha, t)\right\} .
$$

Last,

$$
\begin{gathered}
P_{\alpha}(M, \gamma, t):=H_{\alpha} \cap P(M, \gamma, t) \quad \text { and } \quad C_{\alpha}(M, \gamma, t):=H_{\alpha} \cap C(M, \gamma, t), \\
S_{F H}(M, \gamma):=\bigoplus\left\{\operatorname{SFH}(M, \gamma, \mathfrak{s}): i\left(c_{1}(\mathfrak{s}, t)\right) \in C_{\alpha}(M, \gamma, t)\right\} .
\end{gathered}
$$

For an explanation of the types of well-behaved surfaces mentioned in the last part of this section, see Definitions 2.9 and 2.10. 
If $(M, \gamma)$ is taut and strongly balanced, then $P_{\alpha}(M, \gamma, t)$ is the convex hull of $C_{\alpha}(M, \gamma, t)$ and it is a face of the polytope $P(M, \gamma, t)$. Furthermore, if $S$ is a nice decomposing surface that gives a taut decomposition $(M, \gamma) \stackrel{S}{\rightsquigarrow}\left(M^{\prime}, \gamma^{\prime}\right)$ and $[S]=\alpha$, then $\operatorname{SFH}\left(M^{\prime}, \gamma^{\prime}\right)=\operatorname{SFH}(M, \gamma)$ (see Juhász [19, Proposition 4.12]).

We conclude this section with the following theorem.

Theorem 2.8 (Juhász [19, Corollary 4.15]) Let $(M, \gamma)$ be a taut balanced sutured manifold, and suppose that $H_{2}(M)=0$. Then the following two statements hold.

(i) For every $\alpha \in H_{2}(M, \partial M)$, there exists a groomed surface decomposition

$$
(M, \gamma) \stackrel{S}{\rightsquigarrow}\left(M^{\prime}, \gamma^{\prime}\right)
$$

such that $\left(M^{\prime}, \gamma^{\prime}\right)$ is taut, $[S]=\alpha$ and

$$
\operatorname{SFH}\left(M^{\prime}, \gamma^{\prime}\right) \cong \operatorname{SFH}_{\alpha}(M, \gamma) \text {. }
$$

If moreover $\alpha$ is well-groomed, then $S$ can be chosen to be well-groomed.

(ii) For every face $F$ of $P(M, \gamma, t)$, there exists an $\alpha \in H_{2}(M, \partial M)$ such that $F=P_{\alpha}(M, \gamma, t)$.

\subsection{Well-behaved surfaces}

The result of decomposition along some surfaces can be described more easily than along others. Here we summarise the different types of surfaces, groomed, well-groomed, and nice, as well as how sutured Floer homology behaves under decomposition.

Two parallel curves or arcs $\lambda_{1}$ and $\lambda_{2}$ in a surface $S$ are said to be coherently oriented if $\left[\lambda_{1}\right]=\left[\lambda_{2}\right] \in H_{1}(S, \partial S)$.

Definition 2.9 (Gabai [14, Definition 0.2]) If $(M, \gamma)$ is a balanced sutured manifold, then a surface decomposition $(M, \gamma) \stackrel{S}{\rightsquigarrow}\left(M^{\prime}, \gamma^{\prime}\right)$ is called groomed if for each component $V$ of $R(\gamma)$ one of the following is true:

(i) $S \cap V$ is a union of parallel, coherently oriented, nonseparating closed curves.

(ii) $S \cap V$ is a union of arcs such that for each component $\delta$ of $\partial V$, have $|\delta \cap \partial S|=$ $|\langle\delta, \partial S\rangle|$.

A groomed surface is called well-groomed if for each component $V$ of $R(\gamma)$ it holds that $S \cap V$ is a union of parallel, coherently oriented, nonseparating closed curves or arcs. 
In order to define a nice surface, we need the following definition. A curve $C$ is boundary coherent if either $[C] \neq 0$ in $H_{1}(R ; \mathbb{Z})$, or $[C]=0$ in $H_{1}(R ; \mathbb{Z})$ and $C$ is oriented as the boundary of the component of $R \backslash C$ that is disjoint from $\partial R$.

Definition 2.10 (Juhász [19, Definition 3.22]) A decomposing surface $S$ in $(M, \gamma)$ is called nice if $S$ is open, $v_{0}$ is nowhere parallel to the normal vector field of $S$, and for each component $V$ of $R(\gamma)$ the set of closed components of $S \cap V$ consists of parallel, coherently oriented and boundary coherent simple closed curves.

An important observation is that any open and groomed surface can be made into a nice surface by a small perturbation which places its boundary into a generic position.

Finally we set the groundwork for the notion of "extremal Spinc structure" given in Definition 4.8 .

Definition 2.11 (Juhász [18, Definition 1.1]) Let $(M, \gamma)$ be a balanced sutured manifold and let $(S, \partial S) \subset(M, \partial M)$ be a properly embedded oriented surface. An element $\mathfrak{s} \in \operatorname{Spin}^{c}(M, \gamma)$ is called outer with respect to $S$ if there is a unit vector field $v$ on $M$ whose homology class is $\mathfrak{s}$ and $v_{p} \neq\left(-v_{S}\right)_{p}$ for every $p \in S$. Here $v_{S}$ is the unit normal vector field of $S$ with respect to some Riemannian metric on $M$. Let $O_{S}$ denote the set of outer $\operatorname{Spin}^{c}$ structures.

Theorem 2.12 (Juhász [18, Theorem 1.3]) Let $(M, \gamma)$ be a balanced sutured manifold and let

$$
(M, \gamma) \stackrel{S}{\rightsquigarrow}\left(M^{\prime}, \gamma^{\prime}\right)
$$

be a sutured manifold decomposition along a nice surface $S$. Then

$$
\operatorname{SFH}\left(M^{\prime}, \gamma^{\prime}\right)=\bigoplus_{\mathfrak{s} \in O_{S}} \operatorname{SFH}(M, \gamma)
$$

In particular, if $O_{S}$ contains a single $\operatorname{Spin}^{c}$ structure s such that $\operatorname{SFH}(M, \gamma) \neq 0$, then

$$
\operatorname{SFH}\left(M^{\prime}, \gamma^{\prime}\right)=\operatorname{SFH}(M, \gamma, \mathfrak{s}) .
$$

\subsection{Norms on 3-manifolds}

Here we briefly review the various (semi)norms that have been defined on the second homology group $H_{2}(M, \partial M ; \mathbb{R})$ of a 3-manifold $M$ with boundary. This survey is meant to highlight the geometric nature of the sutured Floer polytope.

Note that we are always given a map $H_{2}(M, \partial M) \rightarrow \mathbb{Z}^{\geq 0}$, which is first extended to a rational-valued map on $H_{2}(M, \partial M ; \mathbb{Q})$ by linearity and then to a real-valued map 
on $H_{2}(M, \partial M ; \mathbb{R})$ by continuity. Finally, in each case some work has to be done to show that the resulting map on the real-valued homology group is indeed a seminorm. In each case, we refer to all three maps by the same symbol, but it is obvious which one we mean.

Thurston [28] defined a seminorm on the homology of a 3-manifold $(M, \partial M)$ with possibly empty boundary. Given a properly embedded, oriented closed surface $S \subset M$, set

$$
\chi_{-}(S):=\sum_{\substack{\text { components } \\ S_{i} \text { of } S}} \max \left\{0,-\chi\left(S_{i}\right)\right\} .
$$

Then the Thurston seminorm is given by the map

$$
\begin{gathered}
x: H_{2}(M, \partial M ; \mathbb{R}) \longrightarrow \mathbb{Z}^{\geq 0}, \\
x(\alpha):=\min \left\{\chi_{-}(S):[S]=\alpha \in H_{2}(M, \partial M)\right\} .
\end{gathered}
$$

The seminorm $x$ is a norm if there exists no subspace of $H_{2}(M, \partial M)$ that is spanned by surfaces of nonnegative Euler characteristic, that is, spheres, annuli and tori. The Thurston seminorm measures the "complexity" of a certain homology class. Thurston showed that some top-dimensional faces of the norm unit ball are fibred. That is, a face $F$ is fibred if there exists a fibration $M \rightarrow S^{1}$ with fibre $\Sigma$ such that the ray $r \cdot[\Sigma]$ for $r \in \mathbb{R}^{\geq 0}$ intersects the unit ball in the interior of $F$. Moreover, all of the rational rays through the interior of $F$ in a similar way represent fibrations of $M$.

Scharlemann [25] generalised the Thurston norm. As before let $(M, \partial M)$ be a given 3-manifold and $S$ a properly embedded surface in $M$. Now let $\beta$ be a properly embedded 1-complex in $M$, and define

$$
\chi_{\beta}(S):=\sum_{\substack{\text { components } \\ S_{i} \text { of } S}} \max \left\{0,-\chi\left(S_{i}\right)+\left|S_{i} \cap \beta\right|\right\} .
$$

Then the generalised Thurston norm is given by the map

$$
\begin{gathered}
x_{\beta}: H_{2}(M, \partial M) \longrightarrow \mathbb{Z}^{\geq 0}, \\
x_{\beta}(\alpha):=\min \left\{\chi_{\beta}(S):[S]=\alpha \in H_{2}(M, \partial M)\right\} .
\end{gathered}
$$

The generalised Thurston norm specialises to the case of sutured manifolds (see Cantwell and Conlon [8] and Scharlemann [25]). In particular, suppose that $(M, \gamma)$ is a sutured manifold, and that $S$ is a properly embedded surface. Then let $n(S)$ denote the absolute value of the intersection number of $\partial S$ and $s(\gamma)$ as elements of $H_{1}(\partial M)$. 
Define

$$
\chi_{-}^{s}(S):=\sum_{\substack{\text { components } \\ S_{i} \text { of } S}} \max \left\{0,-\chi\left(S_{i}\right)+\frac{1}{2} n\left(S_{i}\right)\right\} .
$$

Note that if we take $\beta:=s(\gamma)$, then $\chi_{\beta}(S)=2 \chi_{-}^{s}(S)+\chi(S)$. Similarly to before, the sutured Thurston norm is given by the map

$$
\begin{gathered}
x^{s}: H_{2}(M, \partial M) \longrightarrow \mathbb{Z}^{\geq 0}, \\
x^{s}(\alpha):=\min \left\{\chi_{-}^{s}(S):[S]=\alpha \in H_{2}(M, \partial M)\right\} .
\end{gathered}
$$

The motivation for defining $x^{s}$ comes from looking at the manifold $D M$ obtained by gluing two oppositely oriented copies of $M$ along the boundary, that is,

$$
D M:=(M, \gamma) \cup(-M,-\gamma) / \sim,
$$

where the equivalence relation identifies $R_{+}(\gamma)$ with $R_{+}(-\gamma)$ pointwise in the obvious way. Then $D M$ is referred to as the double of $M$. Similarly, if $S$ is a properly embedded surface in $M$, then $D S$ is the double of $S$ in $D M$. Now, Cantwell and Conlon [8, Theorem 2.3] say that there is a natural "doubling map" $D_{*}: H_{2}(M, \partial M ; \mathbb{R}) \rightarrow H_{2}(D M, \partial D M ; \mathbb{R})$, so that for any $\alpha \in H_{2}(M, \partial M ; \mathbb{R})$,

$$
x^{s}(\alpha)=\frac{1}{2} x\left(D_{*}(\alpha)\right) \text {. }
$$

So far all of the described seminorms have been symmetric. As the sutured Floer polytope is asymmetric in general, the unit balls of these seminorms are certainly not dual to the sutured Floer polytope. Also, in order to talk about the polytope as being dual to the unit ball of a seminorm, we must pick a trivialisation because otherwise the polytope is defined only up to translation in $H^{2}(M, \partial M)$.

Fix a balanced sutured manifold $(M, \gamma)$ and a trivialisation $t \in T(M, \gamma)$. Using the theory developed by Juhász, we define an integer-valued function on $H_{2}(M, \partial M)$, dependent on $t$, that plays the role of the Thurston-type norms in the case of the sutured Floer polytope. First of all, associate an integer $c(S, t)$ to an oriented decomposing surface $S$ in $(M, \gamma)$ (see Juhász [19, Definition 3.16]). This purely geometric invariant is given by

$$
c(S, t):=\chi(S)+I(S)-r(S, t),
$$

where $\chi(S)$ is the Euler characteristic, $I(S)$ generalises the term $-\frac{1}{2} n\left(S_{i}\right)$ in the definition of the sutured seminorm, and $r(S, t)$ is an additional component, which accounts for the dependance of the polytope on the trivialisation $t$.

Any generic oriented decomposing surface $S$ is such that the positive unit normal field $v_{S}$ of $S$ is nowhere parallel to $v_{0}$ along $\partial S$. Denote the components of $\partial S$ by 
$T_{1}, \ldots, T_{k}$; each of the components has an orientation coming from the orientation of $S$. Let $w_{0}$ denote the nowhere zero vector field obtained by projecting $v_{0}$ into $T S$. Further, let $f$ be the positive unit tangent vector field of $\partial S$. For $1 \leq i \leq k$, define the index $I\left(T_{i}\right)$ to be the (signed) number of times $w_{0}$ rotates with respect to $f$ as we go around $T_{i}$. Then set

$$
I\left(T_{i}\right):=\sum_{i=1}^{k} I\left(T_{i}\right) .
$$

Next, let $p\left(v_{S}\right)$ be the projection of $v_{S}$ into $v_{0}^{\perp}$. Observe that $p\left(v_{S}\right) \mid \partial S$ is nowhere zero. For $1 \leq i \leq k$ define $r\left(T_{i}, t\right)$ to be the number of times $p\left(v_{S}\right) \mid \partial T_{i}$ rotates with respect to $r$ as we go around $T_{i}$. Then set

$$
r(S, t):=\sum_{i=1}^{k} r\left(T_{i}, t\right) .
$$

Now, assuming that $H_{2}(M)=0$ and for a fixed $t \in T(M, \gamma)$ we define the function

$$
\begin{aligned}
y_{t} & : H_{2}(M, \partial M) \longrightarrow \mathbb{Z}, \\
y_{t}(\alpha) & :=\min \{-c(S, t): S \text { nice decomposing surface, }[S]=\alpha\} .
\end{aligned}
$$

Remark 2.13 As we noted before, any open groomed surface can be slightly perturbed into a nice surface. Any homology class $\alpha \neq 0$ has a groomed surface representative (see Gabai [14, Lemma 0.7]), however it is not clear that it necessarily has an open groomed representative. Thus we have the condition $H_{2}(M)=0$. We could have relaxed the definition and required each $S$ to satisfy all the conditions of being nice except openness, but it is not clear that this would have been helpful.

If $T$ is a component of $\partial S$ such that $T \not \subset \gamma$ then $I(T)=-\frac{1}{2}|T \cap s(\gamma)|$ (see Juhász [19, Lemma 3.17]). In other words, in this case $-I(T)=\frac{1}{2} n(T)$ which is the second term in the definition of $x^{s}$.

We would like to say that the function $y_{t}$ has some useful properties, such as that it satisfies the triangle inequality and positive homogeneity with respect to the integers. Indeed, as we see in Proposition 2.15, these properties follow from the definitions and from Lemma 2.14 .

Lemma 2.14 (Juhász [19, Corollary 4.11]) Let $(M, \gamma)$ be a taut, strongly balanced sutured manifold such that $H_{2}(M)=0$. Then

$$
c(\alpha, t)=\max \{c(S, t): S \text { a nice decomposing surface, }[S]=\alpha\} .
$$


Proposition 2.15 Let $(M, \gamma)$ be a taut, strongly balanced sutured manifold such that $H_{2}(M)=0$. Fix $t \in T(M, \gamma)$. Then for any $\alpha, \beta \in H_{2}(M, \partial M)$ and any $m \in \mathbb{Z}$, the following hold:

$$
\begin{aligned}
y_{t}(|m| \cdot \alpha) & =|m| \cdot y_{t}(\alpha) \\
y_{t}(\alpha+\beta) & \leq y_{t}(\alpha)+y_{t}(\beta)
\end{aligned}
$$

Proof From Lemma 2.14 it follows that $y_{t}(\alpha)=-c(\alpha, t)$ for any $\alpha \in H_{2}(M, \partial M)$. By definition $c(\alpha, t)=\min \{\langle c, \alpha\rangle: c \in P(M, \gamma, t)\}$ (see Equation (3)), thus

$$
y_{t}(\alpha)=\max \left\{\langle-c, \alpha\rangle: c \in P_{t}\right\}
$$

where we have denoted $P(M, \gamma, t)$ by $P_{t}$.

The first statement of the proposition is obvious. Proving the triangle inequality is also easy, and is identical to the proof given by Juhász [19, Proposition 8.2]:

$$
\begin{aligned}
y_{t}(\alpha+\beta) & =\max \left\{\langle-c, \alpha+\beta\rangle: c \in P_{t}\right\} \\
& =\max \left\{\langle-c, \alpha\rangle+\langle-c, \beta\rangle: c \in P_{t}\right\} \\
& \leq \max \left\{\langle-c, \alpha\rangle: c \in P_{t}\right\}+\max \left\{\langle-c, \beta\rangle: c \in P_{t}\right\}=y_{t}(\alpha)+y_{t}(\beta)
\end{aligned}
$$

This completes the proof.

As before we can extend $y_{t}$ to a rational-valued map on $H_{2}(M, \partial M ; \mathbb{Q})$ by linearity and then to a real-valued map on $H_{2}(M, \partial M ; \mathbb{R})$ by continuity. Thus, for any balanced sutured manifold with $H_{2}(M)=0$ we can define a geometric sutured function

$$
y_{t}: H_{2}(M, \partial M) \longrightarrow \mathbb{R}
$$

such that $y_{t}(r \cdot \alpha)=r \cdot y_{t}(\alpha)$ and $y_{t}(\alpha+\beta) \leq y_{t}(\alpha)+y_{t}(\beta)$ for $r \in \mathbb{R}$ and $\alpha, \beta \in H_{2}(M, \partial M ; \mathbb{R})$.

The following corollary says that $y_{t}$ is actually a (semi)norm for a lot of the oftenstudied sutured manifolds.

Corollary 2.16 Let $(M, \gamma)$ be a taut, strongly balanced sutured manifold such that $H_{2}(M)=0$. If there exists a $t \in T(M, \gamma)$ such that

$$
y_{t}: H_{2}(M, \partial M) \longrightarrow \mathbb{R}^{\geq 0},
$$

then $y_{t}$ is an asymmetric seminorm. In particular, this is the case when $H^{2}(M)=0$. Moreover, the unit ball of the seminorm $y_{t}$ is the dual to the polytope $P(M, \gamma, t)$. Finally, $y_{t}$ is a norm if and only if $\operatorname{dim} P(M, \gamma, t)=b_{1}(M)$. 
The fact that such a $t$ exists follows from a lemma of Juhász [19, Lemma 3.12]. Basically, when there is no torsion in $H_{1}(M)$, then we can choose a trivialisation such that $P(M, \gamma, t)$ contains $0 \in H^{2}(M, \partial M)$. The very last statement in the corollary uses the same argument as in the proof of [19, Proposition 8.2].

Remark 2.17 Juhász [19, Definition 8.1] defines an asymmetric seminorm $y$ whose dual seminorm unit ball is $-P(M, \gamma)$, where $-P(M, \gamma)$ is the centrally symmetric image of $P(M, \gamma)$. Here $P(M, \gamma)$ is the polytope with the centre of mass at $0 \in H^{2}(M, \partial M ; \mathbb{R})$. Specifically he defines

$$
\begin{gathered}
y: H_{2}(M, \partial M ; \mathbb{R}) \longrightarrow \mathbb{R}^{\geq 0}, \\
y(\alpha):=\max \{\langle-c, \alpha\rangle: c \in P(M, \gamma)\} .
\end{gathered}
$$

When $t$ is such that the centre of mass of $P(M, \gamma, t)$ lies at $0 \in H^{2}(M, \partial M)$, then $y_{t}=y$.

\section{Foliations on sutured manifolds}

This section sets up the foliation theory necessary to understand our duality result. It begins with some basic theory of foliations on sutured manifolds; further background reading can be found in the papers of Candel and Conlon [3; 4]. We then proceed to describe the way Gabai [13] constructs taut, finite-depth foliations on any given sutured manifold. This is followed by a description of junctures and spiral staircase neighbourhoods, which are used in proving one direction of Lemma C. The last section introduces the foliation cones of Cantwell and Conlon [10].

\subsection{Foliations on sutured manifolds}

Definition 3.1 A foliation $\mathcal{F}$ of a sutured manifold $(M, \gamma)$ is a foliation of $M$ such that the leaves of $\mathcal{F}$ are transverse to $\gamma$ and tangential to $R(\gamma)$ with normal direction pointing inward along $R_{-}(\gamma)$ and outward along $R_{+}(\gamma)$.

The collection of simple closed curves $\partial \gamma \subset \partial M$ can be considered as a set of convex corners on $M$. Elsewhere in the literature, when $M$ is any given manifold, the notation $\partial_{\pitchfork} M$ is used to denote the subset of $\partial M$ where the leaves of $\mathcal{F}$ are transverse to the boundary of $M$, and $\partial_{\tau} M$ to denote the complement $\partial M \backslash \partial_{\pitchfork} M$ where the leaves of $\mathcal{F}$ are tangential to the boundary of $M$.

Let $L$ be a leaf of a foliation $\mathcal{F}$ and let $\left\{C_{\alpha}\right\}_{\alpha \in \mathcal{A}}$ denote the family of all compact subsets of $L$. Let $W_{\alpha}:=L \backslash C_{\alpha}$ and denote by $\bar{W}_{\alpha}$ the closure of $W_{\alpha}$ in $M$. Recall 
that the asymptote of $L$ is the $\operatorname{limit} \operatorname{set} \lim L:=\bigcap_{\alpha \in \mathcal{A}} \bar{W}_{\alpha}$. The asymptote for a noncompact leaf $L$ is a compact, nonempty, $\mathcal{F}$-saturated set, that is, $\lim L$ is a compact set which is a nonempty union of leaves of $\mathcal{F}$. Further, $L$ is said to be proper if it is not asymptotic to itself, and totally proper if every leaf in the closure $\bar{L}$ is a proper leaf.

A leaf $L$ of a foliation $\mathcal{F}$ is said to be at depth 0 if it is compact. For $k>1$, a leaf $L$ is said to be at depth $k$ if $\bar{L} \backslash L$ is a collection of leaves at depths less than $k$, with at least one leaf at depth $k-1$ (see Candel and Conlon [3, Definition 8.3.14]). Note that this definition of depth assumes that the leaf is totally proper. A depth- $k$ foliation is a foliation with all leaves at depth $l \leq k$ and at least one leaf at depth $l=k$.

We are primarily interested in taut depth-one foliations $\mathcal{F}$ of a sutured manifold $(M, \gamma)$. As is described below, these foliations are built from the product foliation $\mathcal{P}$ of a product sutured manifold $\left(M^{\prime}, \gamma^{\prime}\right):=(\Sigma \times I, \partial \Sigma \times I)$. It is sometimes necessary to specify a particular leaf of $\mathcal{P}$, and so we assume that $\mathcal{P}$ is precisely the foliation with leaves $L_{t}:=\Sigma \times t$, for $t \in I=[0,1]$.

Definition 3.2 A transversely oriented codimension one foliation $\mathcal{F}$ on a sutured manifold $(M, \gamma)$ is taut if there exists a curve or properly embedded arc in $M$ that is transverse to the leaves of $\mathcal{F}$ and that intersects every leaf of $\mathcal{F}$ at least once.

Convention 3.3 Let $(M, \gamma)$ be a sutured manifold. From now on, when we say a depth-one or depth-zero foliation of $(M, \gamma)$, it is implicit that these foliations are smooth, transversely oriented, and in the case of the depth-one their sole compact leaves are the connected components of $R(\gamma)$.

Remark 3.4 Suppose that $M$ is a manifold with boundary that fibres over $S^{1}$. Then $\partial M$ is a (possibly empty) collection of tori. If $M$ is made into a sutured manifold by specifying the sutures $\gamma$, then one of two situations occurs:

(i) $\gamma=T(\gamma)=\partial M$ and the fibration is a depth-zero foliation.

(ii) $A(\gamma) \neq \varnothing$, and the fibres of the fibration are not transverse to $\gamma$ or not tangential to $R(\gamma)$.

Set $M_{0}:=M \backslash R(\gamma)$. The following lemma summarises useful information about depth-one foliations.

Lemma 3.5 (Candel and Conlon [4, Lemma 11.4.4]) Let $\mathcal{F}$ be a transversely oriented, $C^{\infty}$ foliation of the connected sutured manifold $(M, \gamma)$, transverse to $\gamma$ and having the components of $R(\gamma)$ as sole compact leaves. Let $\mathcal{L}$ be a smooth onedimensional foliation transverse to $\mathcal{F}$ and tangent to $\gamma$, so that $\mathcal{L} \mid A(\gamma)$ is a foliation by compact, properly embedded arcs. Then the following statements are equivalent. 
(i) $\mathcal{F}$ is a taut depth-one foliation.

(ii) There is a smoothly embedded circle $\Sigma \subset M_{0}$ that is transverse to $\mathcal{F} \mid M_{0}$, meeting each leaf of that foliation exactly once.

(iii) $\mathcal{L}$ can be chosen to have a closed leaf in $M_{0}$ that meets each leaf of $\mathcal{F} \mid M_{0}$ exactly once.

(iv) $\mathcal{F} \mid M_{0}$ fibres $M_{0}$ over $S^{1}$. In this case, there is a $C^{0}$ flow $\Phi_{t}$ on $M$ having the leaves of $\mathcal{L}$ as flow lines, stationary at the points of $R(\gamma)$, smooth on $M_{0}$ and carrying the leaves of $\mathcal{F}$ diffeomorphically onto one another.

Let $\mathcal{F}$ be a depth-one foliation on $(M, \gamma)$. Then $\mathcal{F}$ determines a fibration $M_{0} \rightarrow S^{1}$, with fibres the noncompact leaves of $\mathcal{F}$.

Following Cantwell and Conlon [9, Section 2], we associate to each depth-zero or depth-one foliation a cohomology class $H^{1}(M)$ using Lemma 3.5. In particular, the fibration of $M_{0}$ over $S^{1}$ gives rise to a map

$$
\lambda(\mathcal{F}): \pi_{1}(M) \longrightarrow \mathbb{Z}=\pi_{1}\left(S^{1}\right),
$$

which passes to a cohomology class $\lambda(\mathcal{F}) \in H^{1}(M)$.

Remark 3.6 Note that it follows from the definition that $\lambda(\mathcal{F})$ is a nondivisible class.

Definition 3.7 Two depth-zero or depth-one foliations $\mathcal{F}$ and $\mathcal{F}^{\prime}$ are said to be equivalent, denoted by $\mathcal{F} \sim \mathcal{F}^{\prime}$, if $\mathcal{F}$ is isotopic to $\mathcal{F}^{\prime}$ via a continuous isotopy that is smooth in $M_{0}$.

The following theorem says that an equivalence class of foliations $\mathcal{F}$ is uniquely determined by $\lambda(\mathcal{F})$.

Theorem 3.8 (Cantwell and Conlon [9, Theorem 1.1]) Let $\mathcal{F}$ and $\mathcal{F}^{\prime}$ be depth-one type foliations of $(M, \gamma)$, such that $\lambda(\mathcal{F})=\lambda\left(\mathcal{F}^{\prime}\right)$. Then $\mathcal{F}$ is equivalent to $\mathcal{F}^{\prime}$.

Note that Theorem 3.8 is also true when the foliations are of depth zero, that is, when they are fibrations of $M$ with fibres transverse to $\gamma$. 


\subsection{Gabai's construction of depth-one foliations}

In the well-known paper [13], Gabai gives a way of constructing finite depth foliations on sutured manifolds from a sutured manifold hierarchy. A sutured manifold hierarchy is a sequence of decompositions along surfaces

$$
\left(M_{0}, \gamma_{0}\right) \stackrel{S_{1}}{\rightsquigarrow}\left(M_{1}, \gamma_{1}\right) \stackrel{S_{2}}{\rightsquigarrow} \ldots \stackrel{S_{n}}{\rightsquigarrow}\left(M_{n}, \gamma_{n}\right)
$$

where $\left(M_{n}, \gamma_{n}\right)$ is a product sutured manifold.

Theorem 3.9 (Gabai [13, Theorem 5.1]) Suppose $M$ is connected and $(M, \gamma)$ has a sutured manifold hierarchy

$$
(M, \gamma)=\left(M_{0}, \gamma_{0}\right) \stackrel{S_{1}}{\rightsquigarrow}\left(M_{1}, \gamma_{1}\right) \stackrel{S_{2}}{\rightsquigarrow} \ldots \stackrel{S_{n}}{\rightsquigarrow}\left(M_{n}, \gamma_{n}\right)
$$

so that no component of $R\left(\gamma_{i}\right)$ is a compressing torus. Then there exist transversely oriented foliations $\mathcal{F}$ and $\mathcal{G}$ of $M$ such that the following hold.

(i) $\mathcal{F}$ and $\mathcal{G}$ are tangent to $R(\gamma)$.

(ii) $\mathcal{F}$ and $\mathcal{G}$ are transverse to $\gamma$.

(iii) If $H_{2}(M, \gamma) \neq 0$, then every leaf of $\mathcal{F}$ and $\mathcal{G}$ nontrivially intersects a transverse closed curve or a transverse arc with endpoints in $R(\gamma)$. However, if $\varnothing \neq \partial M=$ $R_{+}(\gamma)$ or $R_{-}(\gamma)$, then this holds only for interior leaves.

(iv) There are no 2-dimensional Reeb components of $\mathcal{F} \mid \gamma$ and $\mathcal{G} \mid \gamma$.

(v) $\mathcal{G}$ is $C^{\infty}$ except possibly along toroidal components of $R(\gamma)$ or along toroidal components of $S_{1}$ if $\partial M=\varnothing$.

(vi) $\mathcal{F}$ is of finite depth.

The ideas from this proof have been exploited a lot; a good summary of the construction is given by Calegari [2, Section 5.6]. However, understanding the construction in the case when $n=1$ is essential for the proof of Lemma $\mathrm{C}$, so we explain the ideas in this simplified case.

Let $(M, \gamma)$ be a sutured manifold with a properly embedded surface $S$ in $M$, such that $(M, \gamma) \stackrel{S}{\rightsquigarrow}\left(M^{\prime}, \gamma^{\prime}\right)$ gives a product manifold $\left(M^{\prime}, \gamma^{\prime}\right)$. Gabai's construction proceeds as follows: first, he shows that there is a series of decompositions along well-groomed surfaces $S_{i} \subset M_{i-1}$ giving the same product manifold

$$
(M, \gamma) \stackrel{S_{1}}{\rightsquigarrow}\left(M_{1}, \gamma_{1}\right) \stackrel{S_{2}}{\rightsquigarrow} \ldots \stackrel{S_{n}}{\rightsquigarrow}\left(M^{\prime}, \gamma^{\prime}\right)
$$

second, he demonstrates how to produce a $C^{0}$ foliation $\mathcal{F}_{k-1}$ on $\left(M_{k-1}, \gamma_{k-1}\right)$ from a finite-depth $C^{0}$ foliation $\mathcal{F}_{k}$ on $\left(M_{k}, \gamma_{k}\right)$, such that $\operatorname{depth}\left(\mathcal{F}_{k-1}\right)=\operatorname{depth}\left(\mathcal{F}_{k}\right)+1$. 
This means that if $S$ is well-groomed, starting from the product foliation on $\left(M^{\prime}, \gamma^{\prime}\right)$ we have a recipe how to construct a taut depth-one $C^{0}$ foliation $\mathcal{F}=\mathcal{F}_{0}$ on $(M, \gamma)$.

Remark 3.10 When we have $(M, \gamma) \stackrel{S_{1}}{\rightsquigarrow \rightarrow}\left(M_{1}, \gamma_{1}\right)$ for a product manifold $\left(M_{1}, \gamma_{1}\right)$, then the construction of $\mathcal{F}$ coincides with the construction of $\mathcal{G}$. This means that $\mathcal{F}$ is $C^{\infty}$ except possibly along toroidal components. However, as we are starting from the product foliation on $\left(M_{1}, \gamma_{1}\right)$, actually $\mathcal{F}$ is smooth along toroidal components as well.

So far we have that if $S$ is well-groomed the "only if" direction of Lemma C follows straight from Theorem 3.9. Thus, the content of the proof of the "only if" direction of Lemma $\mathrm{C}$ below is the removal of the condition for $S$ to be well-groomed. When we remove this condition in Lemma 4.2, we do not use Gabai's method of showing that any decomposition can be broken down into the well-groomed ones. Thus we omit the details of this first step in his proof; see Gabai [13, Theorem 5.4]. However, we explain the remaining steps of his construction, as it is crucial to the rest of the paper.

Let us focus on constructing $\mathcal{F}$ from a product foliation $\mathcal{P}$ on $\left(M^{\prime}, \gamma^{\prime}\right)$ when $S$ is well-groomed and nonempty. Let $V$ be a component of $R(\gamma)$. Recall that $S$ being well-groomed is a condition on $\partial S \cap V$; in particular, that $\partial S \cap V$ is a (possibly empty) collection of parallel, coherently oriented, nonseparating closed curves or arcs. The construction describes a process of spinning $S$ near $V$. It suffices to show how this process works near $V$, as it is analogous for all other components.

Consider each of the following cases: first, $\partial S \cap V$ is empty; second, $\partial S \cap V$ is a collection of closed curves; third, $\partial S \cap V$ is a collection of arcs. For definiteness assume that $V \subset R_{+}(\gamma)$.

Case 1 Suppose that $\partial S \cap V$ is empty for some component $V$. Then $V$ is a component of $R_{+}\left(\gamma^{\prime}\right)$, but since $\left(M^{\prime}, \gamma^{\prime}\right)$ is a product manifold $(\Sigma \times I, \partial \Sigma \times I)$, it follows that $V=R_{+}\left(\gamma^{\prime}\right)$. As $S_{+}$must be contained in $R_{+}\left(\gamma^{\prime}\right)$, it follows that $S=\varnothing$, which is a contradiction. An analogous argument works for $V \subset R_{-}(\gamma)$. Thus, either $\partial S \cap V \neq \varnothing$ for all components $V \subset R(\gamma)$ or $R(\gamma)=\varnothing$. In the latter case $\gamma=T(\gamma)$ and $R_{ \pm}\left(\gamma^{\prime}\right) \cong S_{ \pm}$. Then $M$ fibres over $S^{1}$ with fibre homeomorphic to $S$ and $\mathcal{F}$ is the fibration.

Case 2 Suppose that $\partial S \cap V$ is a collection of parallel, coherently oriented, nonseparating closed arcs. Denote by $\mathcal{J}$ the collection of arcs of $\partial S \cap V$ and label the $\operatorname{arcs} J_{1}, \ldots, J_{l}$ starting with one outermost arc, and proceeding in the obvious way. Section 3.3 gives the motivation for using the letter $J$, which stands for juncture. We first explain how to construct $\mathcal{F}$ near $V$ when $l=1$. It is then fairly clear how to extend the construction to the general case. 


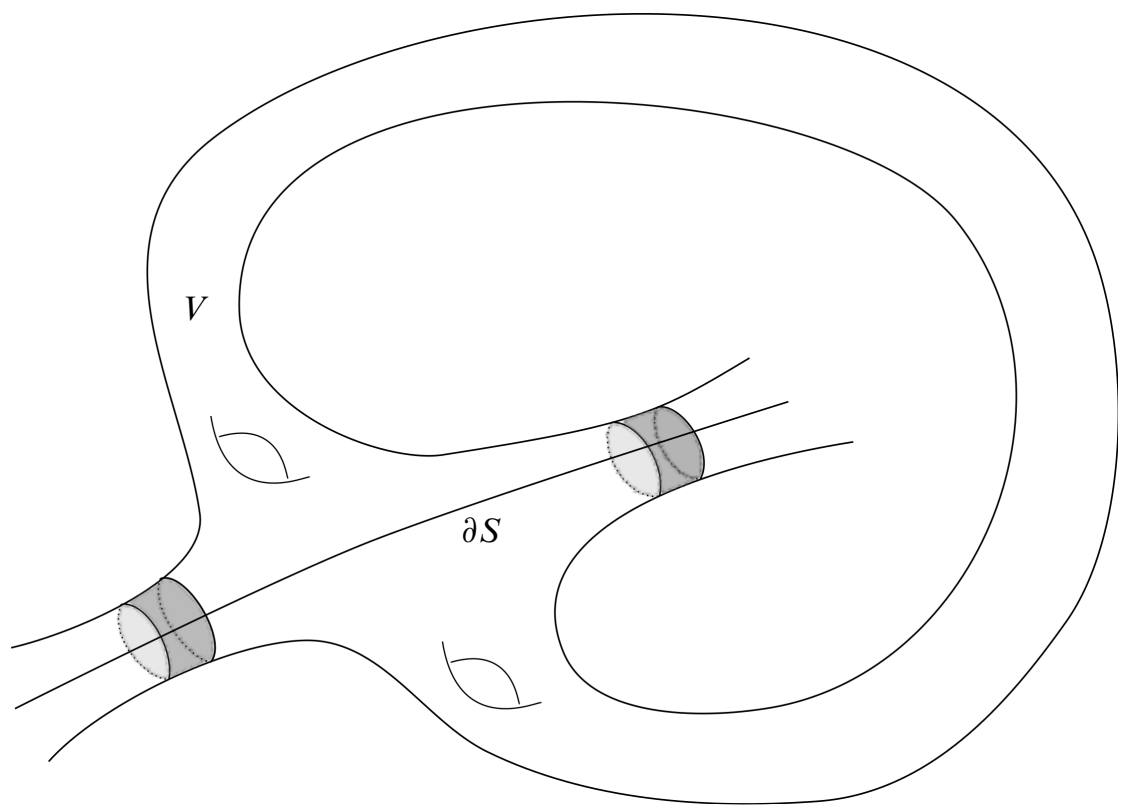

Figure 1: Example where $\partial S \cap V$ is a single arc: the shaded annuli are two annular components of $\gamma$.

Suppose that $\partial S \cap V$ is a single arc $J$; for an example see Figure 1. When decomposing along $S$, we remove a neighbourhood $N(S) \cong S \times I$, where $S \times \partial I=S_{+} \cup S_{-}$; see Definition 2.5 for the labelling convention. Define $J^{+}:=S_{+} \cap V$ and $J^{-}:=S_{-} \cap V$. Consider a neighbourhood $N:=J \times(-2,0)$ of $J$ in $V$, parametrised so that $J^{-}=J \times 0$ and $J^{+}=J \times-1$ (Figure 2). The reason for parametrising an interval as $(-2,0)$ becomes clear shortly.

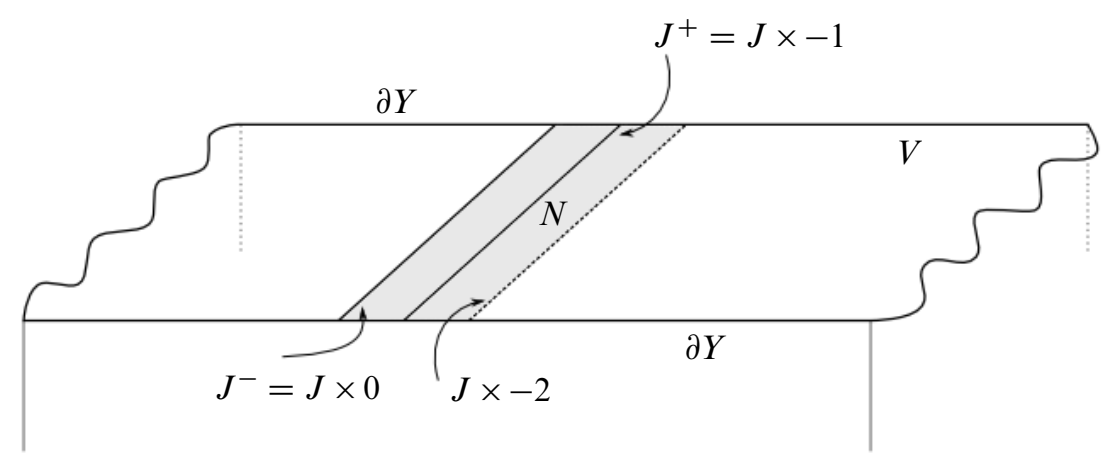

Figure 2: A parametrised neighbourhood of $\partial S \cap V=J$ 
Start with the product foliation $\mathcal{P}$ on $\left(M^{\prime}, \gamma^{\prime}\right)$. Glue $\left(M^{\prime}, \gamma^{\prime}\right)$ along $S_{+}$and $S_{-}$to recover $(M, \gamma)$, in such a way that $J^{+}$is a concave corner, whereas $J^{-}$is a convex corner in $V$ (Figure 3).

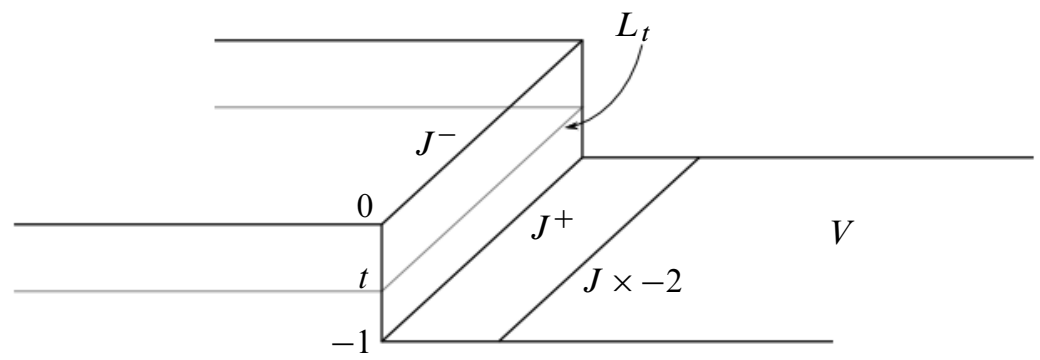

Figure 3: A leaf $L_{t}$ of the product foliation $\mathcal{P}$ is transverse to $V$ after the regluing of $S_{+}$and $S_{-}$.

Let $K$ be the product manifold $(V \backslash N) \times[0, \infty]$ with the product foliation; that is, the leaves are of the form $(V \backslash N) \times s$, for $s \in[0, \infty]$. The manifold obtained by gluing $K$ to $M$ in the obvious way,

$$
M \cup_{(V \backslash N) \sim(V \backslash N) \times 0} K,
$$

is homeomorphic to $M$, but it has a "ditch" with two "walls" $W_{0}$ and $W_{1}$ (Figure 4). Note that $W_{0}=J \times-1 \times[-1, \infty]$ and $W_{1}=J \times-2 \times[0, \infty]$. It is now evident why we chose the parametrisation $(-2,0)$ : so that the last coordinate of $W_{0}$ is parametrised as $[-1, \infty]$.

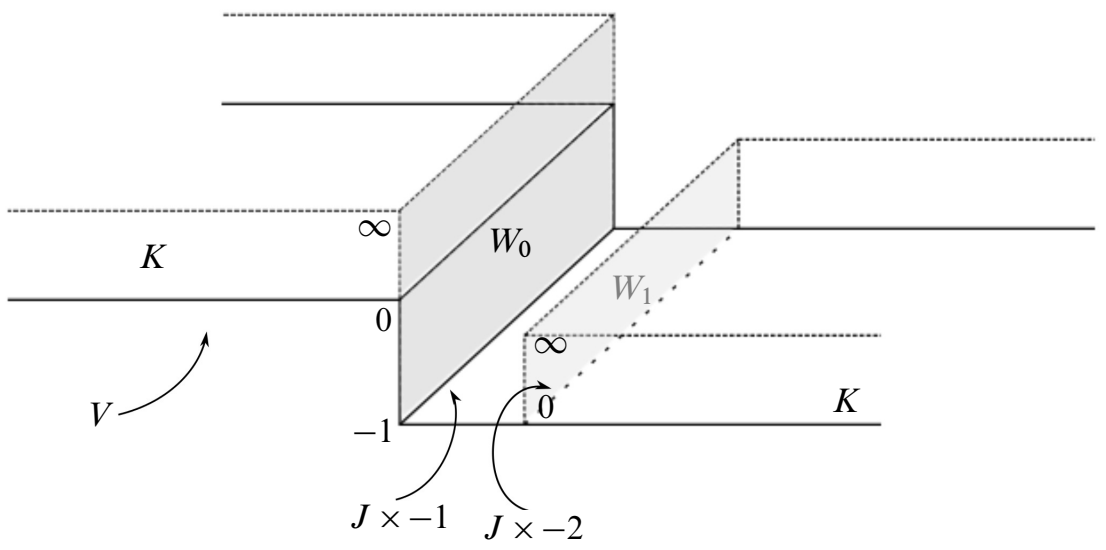

Figure 4: The manifold $M \cup K \cong M$ 
There is now a foliation $\mathcal{F}^{\prime}$ of $M \cup K$ obtained from $\mathcal{P}$ union the product foliation on $K$, however it is not a foliation of the sutured manifold $(M, \gamma)$. The leaves of $\mathcal{F}^{\prime}$ are transverse to the walls and tangential everywhere else on $V$. It remains to glue a product manifold $D$ parametrised as $J \times[-2,-1] \times[0, \infty]$ with a product foliation whose leaves are parallel to $J \times[-2,-1]$. The gluing is done by making pointwise identifications:

(i) $W_{0} \sim J \times-1 \times[0, \infty]$ via the equivalence relation

$$
(x,-1, t) \sim(x,-1, t+1)
$$

(ii) $J \times-2 \times[0, \infty] \sim W_{1}$ via the (identity) equivalence relation

$$
(x,-2, t) \sim(x,-2, t)
$$

So the ditch has been filled in with a product neighbourhood in such a way that the new foliation $\mathcal{F}$ is of depth-one and is tangent to $V$.

Note that the leaf $L_{t} \subset \mathcal{P}$ from Figure 3 has now become a leaf that "spirals" onto $V$. See Figure 5 for a two-dimensional representation of what $L_{t}$ looks like near $J$ as it spirals onto $V$.

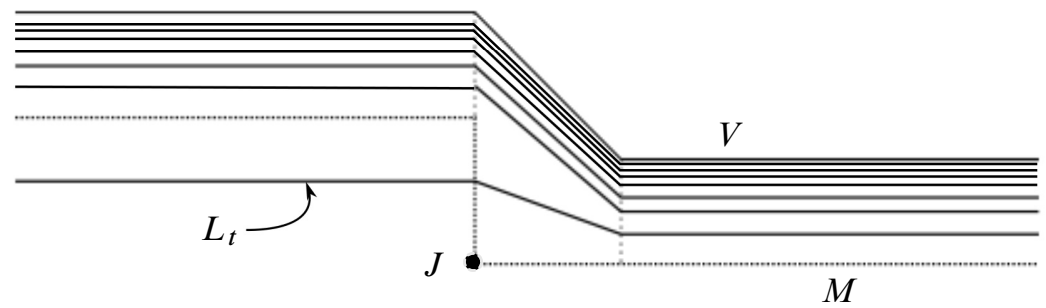

Figure 5: A depth-one leaf $L_{t}$ of the $\mathcal{F}$ spiralling onto $V$

The identification (9) ensures that two interior leaves $L_{t}$ and $L_{s}$ of $\mathcal{P}$ are spun onto $V$ in such a way that their distance along a transverse arc remains the same, and hence there can be no holonomy along the noncompact leaves. This means that we have constructed the (depth-one) foliation $\mathcal{F}$ of $(M, \gamma)$. The manifold $K \cup J \times[-2,-1] \times[0, \infty]$ is a so called spiral staircase neighbourhood of $V$ for the foliation $\mathcal{F}$ as is explained in Section 3.3.

In the general case, since the construction takes place in a collar neighbourhood of a component of $R(\gamma)$, it can be repeated for any number of components $V$ for which $\partial S \cap V$ is nonempty and the intersection consists of arcs. Further, if $\mathcal{J}=\partial S \cap V$ 
contains $l>1$ arcs, then a simple modification of the above construction yields a depthone foliation $\mathcal{F}$ of $(M, \gamma)$. More specifically, it suffices to replace $J$ by $\bigcup_{i=1}^{l} J_{i}$ and to repeat the same gluings of the walls in each of the $l$ ditches $D_{i}$, labeled after their associated junctures $J_{i}$. The key observation is that since the arcs are well-groomed and the identification in (9) is specified to induce no holonomy, the depth of each noncompact leaf is one. Then the spiral staircase neighbourhood becomes a "staircase" indeed, with $l$ steps.

Case 3 Suppose that $\partial S \cap V$ is a collection of parallel, coherently oriented, nonseparating closed curves. The same construction as described above works, only the $J_{i}$ are now going to be parallel closed curves, as opposed to arcs.

Remark 3.11 The described construction slightly differs from the one given by Gabai originally in [13, Theorem 5.1]. For closed curves instead of gluing a copy of $\left(V \backslash \bigcup_{i} J_{i} \times(-1,1)\right) \times[0, \infty]$, he finishes off the foliation using a " $\frac{1}{2}$ infinity cover" of $V$. Here it is more convenient to use the "creating ditches" method in both cases, as it is easier to see why the well-groomed condition can be removed.

In the notation of [13, Theorem 5.1], the foliations $\mathcal{F}_{0}$ (constructed to be of finite depth) and $\mathcal{F}_{1}$ (constructed to be smooth) are identical and of depth-one after the first step of their constructions when starting from a product manifold. The construction we give here also yields the same foliation and is therefore smooth.

From now on we refer to the construction described in this section as Gabai's construction.

\subsection{Junctures and spiral staircases}

In the previous section we discussed Gabai's method for constructing a taut depth-one foliation $\mathcal{F}$ from a well-groomed surface decomposition $(M, \gamma) \rightsquigarrow\left(M^{\prime}, \gamma^{\prime}\right)$ that results in a product $\left(M^{\prime}, \gamma^{\prime}\right)$. This construction is crucial in proving the "only if" direction of Lemma $\mathrm{C}$. The connected components of the 1-manifold $\partial S \cap \partial M$ are called the junctures of the foliation $\mathcal{F}$. We now explain the role that junctures play in the spiralling of leaves of a depth-one foliation along the components of $R(\gamma)$, as understanding this is crucial in proving the "if" direction of Lemma C. The following terminology and theory can be found in Candel and Conlon [3] and Cantwell and Conlon [5, Section 2].

Let $\left\{K_{\alpha}\right\}_{\alpha \in A}$ be the family of all compact subsets of $L$, and let $\left\{U_{\alpha}\right\}_{\alpha \in A}$ be the family of sets $U_{\alpha}:=L \backslash K_{\alpha}$. Then consider descending chains $\left\{U_{\alpha_{i}}\right\}_{i=1}^{\infty}$ of the form

$$
U_{\alpha_{1}} \supsetneq U_{\alpha_{2}} \supsetneq \cdots \supsetneq U_{\alpha_{n}} \supsetneq \cdots
$$


that satisfy the condition

$$
\bigcap_{i=1}^{\infty} U_{\alpha_{k}}=\varnothing .
$$

Two such chains, $\mathcal{U}:=\left\{U_{\alpha_{i}}\right\}_{i=1}^{\infty}$ and $\mathcal{V}:=\left\{V_{\beta_{i}}\right\}_{i=1}^{\infty}$ are related, and their relation denoted by $\mathcal{U} \sim \mathcal{V}$, if for each $i \geq 1$ there exists an $n>i$ such that

$$
U_{\alpha_{i}} \supset V_{\beta_{n}} \text { and } V_{\beta_{i}} \supset U_{\alpha_{n}} .
$$

Clearly this is an equivalence relation. An equivalence class of such descending chains is called an end of $L$. Any descending chain $\mathcal{U}$ that belongs to the equivalence class of an end $e$ is said to be a fundamental open neighbourhood system of $e$. If an open set $U \subset L$ contains a fundamental open neighbourhood system of $e$, then $U$ is said to be a neighbourhood of $e$. Denote by $\mathcal{E}(L)$ the set of ends of $L$.

Let $f: L \rightarrow L$ be a homeomorphism. Then an end $e$ of $L$ is said to be cyclic of period $p$ if $f^{p}(e)=e$ and $p$ is the least such integer. An end $e$ is called attracting (repelling) if there is a neighbourhood $U$ of $e$ and an integer $n>0(n<0)$ such that $f^{n}(\bar{U}) \subset U$ and $\bigcap_{i=1}^{\infty} f^{n}(U)=\varnothing$, where $\bar{U}$ denotes the closure of $U$ in $L$. Attracting and repelling ends are called periodic ends.

It is clear that periodic ends are cyclic, but the converse is not true in general; see Cantwell and Conlon [5, page 4]. If a homeomorphism $f: L \rightarrow L$ is such that all cyclic ends are periodic, then $f$ is called an endperiodic homeomorphism of $L$.

Lemma 3.12 Let $\mathcal{F}$ be a taut depth-one foliation of the sutured manifold $(M, \gamma)$. Then every depth-one leaf $L$ has finitely many ends. Hence, for any homeomorphism $f: L \rightarrow L$ every end $e \in \mathcal{E}(L)$ is cyclic.

Proof Let $\pi: M_{0} \rightarrow S^{1}$ be the fibration induced by $\mathcal{F}$; see Lemma 3.5. For every component $\delta$ in $\gamma$, the foliation $\mathcal{F}$ induces on $\delta$ either a fibration or a depth-one foliation $\mathcal{F}_{\delta}$ with two compact leaves and noncompact leaves homeomorphic to the open interval $(0,1)$. The number of ends of a noncompact leaf $L$ is equal to the number of components of $L \cap \delta$ summed over all $\delta \subset \gamma$ when $\mathcal{F}_{\delta}$ is of depth-one. Note that if $\delta \subset T(\gamma)$ then $\mathcal{F}_{\delta}$ is a fibration.

Let $\delta \subset A(\gamma)$ and so $\mathcal{F}_{\delta}$ is of depth-one. Let $a$ be an arc transverse to $\partial \delta$ with one endpoint in each component of $\partial \delta$. Let $l$ be a depth-one leaf of $\mathcal{F}_{\delta}$. Then $l \cap a$ is a collection of points $\mathcal{A}$ with limit points at $\partial a$. Choose two points $x, y \in \mathcal{A}$, so that the line segment from $x$ to $y$ in $a$, denoted by $x y$, contains no other points of $\mathcal{A}$. Then any other leaf $l^{\prime}$ of $\mathcal{F}_{\delta}$ intersects $x y$ in precisely one point. So if a noncompact leaf $L$ of $\mathcal{F}$ intersects $\delta$ in infinitely many leaves of $\mathcal{F}_{\delta}$, then $|\partial L \cap x y|$ is also an infinite 
collection of points. As $x y$ is a compact interval, the points $\{\partial L \cap x y\}$ have a limit point in the interval $x y$, thus contradicting the fact that $\mathcal{F}_{\delta}$ is of depth-one.

Let $\mathcal{L}$ be a 1 -dimensional foliation transverse to a taut depth-one foliation $\mathcal{F}$ of a sutured manifold $(M, \gamma)$. All smooth, codimension-1 foliations $\mathcal{F}$ admit at least one such transverse foliation $\mathcal{L}$. If $L$ is a depth-one leaf, define $f: L \rightarrow L$ to be the first return map given by $\mathcal{L}_{f}:=\mathcal{L}$. Then $f$ is endperiodic, that is, all of the (cyclic) ends of $L$ are periodic (see Fenley [11]). In combination with Lemma 3.12 this means that all ends of a taut depth-one foliation are periodic.

Next, we review some terminology related to "spiralling" in of leaves and ends. Fix a depth-one leaf $L$. As before let $K_{\alpha}$ be the family of all compact subsets of $L$. Let $U_{\alpha}:=L \backslash K_{\alpha}$, and denote by $\bar{U}_{\alpha}$ the closure of $U_{\alpha}$ in $M$. Then the set $\lim L:=\bigcap_{\alpha \in A} \bar{U}_{\alpha}$ is a compact, nonempty, $\mathcal{F}$-saturated set (see Candel and Conlon [3, Lemma 4.3.2]). Thus we say that $L$ is asymptotic to a leaf $F$ if $F \subset \lim L$.

Similarly, if $\mathcal{U}:=\left\{U_{\alpha_{i}}\right\}_{i=1}^{\infty}$ is a fundamental open neighbourhood system of an end $e$ of $L$, and $\bar{U}_{\alpha_{i}}$ the closure of $U_{\alpha_{i}}$ in $M$, then we have $\lim _{e} L:=\bigcap_{i=1}^{\infty} \bar{U}_{\alpha_{i}}$. Again, [3, Lemma 4.3.5] says that for each leaf $L$ of $\mathcal{F}$ and each end $e \in \mathcal{E}(L)$, the set $\lim _{e} L$ is a compact, nonempty $\mathcal{F}$-saturated set, not depending on the choice of fundamental neighbourhood system of $e$. Thus we also say that the end $e$ is asymptotic to a leaf $F \subset \lim _{e} L$.

Finally, we arrive at the definition of junctures. Let $e$ be an attracting, cyclic end of $L$ of period $n_{e}$, and let $\mathcal{U}=\left\{U_{i}\right\}_{i=1}^{\infty}$ be a fundamental open neighbourhood system. Define $\bar{U}_{i}$ to be the closure of $U_{i}$ in $L$. From the definition of a neighbourhood of $e$, we know that the closure of $\partial U_{i} \backslash \partial L$ is a compact 1 -manifold that separates $L$. It follows that the sets $B_{i}:=\bar{U}_{i} \backslash U_{i+1}$ are closed, not necessarily compact, subsurfaces of $L$, such that $\partial B_{i} \backslash \partial L$ is also a compact 1-manifold. Moreover, since the end is cyclic, it follows that $f^{n_{e}}\left(B_{i}\right)=B_{i+1}$ and that $\bar{U}_{i}=B_{i} \cup B_{i+1} \cup \cdots$ for all $i \geq 0$. The subsurfaces $B_{i}$ are called fundamental domains for the attracting end.

Definition 3.13 The compact 1 -manifolds $J_{i}:=\bar{U}_{i} \backslash U_{i}$ for $0 \leq i<\infty$ are called positive (negative) junctures for an attracting (repelling) end $e$.

Note that the connected components of junctures can be both closed curves and properly embedded arcs.

Actually, one can also define a set of curves on $R(\gamma)$ called junctures. Let $V$ be a component of $R(\gamma)$. As before, suppose that $\mathcal{F}$ is a taut depth-one foliation of $(M, \gamma)$, so certainly $V$ is a compact leaf of $\mathcal{F}$. Let $e$ be an end of a depth-one leaf $L$ 
which is asymptotic to $V$. Then if $f: L \rightarrow L$ is the first return map, it follows from Lemma 3.12 that $f$ is endperiodic with some period $n_{e}$. Therefore, as above, we can find a set of junctures $J_{i}$ starting from a neighbourhood $U$ of $e$. As $f$ is the first return map defined by a transverse 1-dimensional foliation $\mathcal{L}_{f}$, flowing along $\mathcal{L}_{f}$ each $J_{i}$ is carried into $J_{i+t}$ at time $t \in \mathbb{N}$. More specifically, $f$ defines a semimap (local homeomorphism with path lifting property) $p: U \rightarrow V$ which takes each $B_{i}$ (locally) onto $V$, and which maps each $J_{i}$ to $J_{i+1}$. Therefore, $J_{V}:=p\left(J_{i}\right)$ for $i \geq 0$ is a well-defined 1 -manifold in $V$, and is also referred to as a juncture of $\mathcal{F}$ in the component $V$ of $R(\gamma)$. Note that the construction of the juncture depends on the choice of the fundamental neighbourhood system $\mathcal{U}$.

Using junctures one may easily describe the behaviour of a taut depth-one foliation near a compact leaf. As always, let $V$ be a component of $R(\gamma)$. Then there exists a spiral staircase (neighbourhood) $\mathcal{N}_{V}$ associated to $\mathcal{F}$ and $\mathcal{L}_{f}$ (see Cantwell and Conlon [5, Section 12.2.2]). Again suppose that $L$ is a noncompact leaf of $\mathcal{F}$, and $e$ is an end of $L$ that is asymptotic to $V$. For the sake of definiteness suppose that $e$ is an attracting end. Using the same notation as before, suppose that $\mathcal{U}$ is a fundamental neighbourhood system of $e$, and for some $i, J_{i}$ is a positive juncture for $e$. Then there is a surface $T_{i} \cong J_{i} \times[0,1]$ transverse to $\mathcal{F}$ such that for each point $x \in J_{i}$, the arc $x \times[0,1] \in T_{i}$ is contained in a flowline of $\mathcal{L}_{f}$. Note that $T_{i} \subset T_{i+1}$. The surface $B_{i} \cup T_{i}$ separates $M$ into two connected components; the component which contains $V$ is called a spiral staircase neighbourhood denoted by $\mathcal{N}_{V}$, or denoted by $\mathcal{N}_{V}^{\mathcal{U}, i}$ if we wish to emphasise the choice of $\mathcal{U}$ and $i$. Observe that $\mathcal{N}_{V}^{\mathcal{U}, i+1} \subset \mathcal{N}_{V}^{\mathcal{U}, i}$, so the choice of $i$ can be seen as affecting the "size" of the neighbourhood; in other words, any given collar neighbourhood of $V$ contains $\mathcal{N}_{V}^{\mathcal{U}, i}$ for any choice of $\mathcal{U}$ and for all $i>i_{0}$, for some $i_{0}$ large enough.

\subsection{Foliation cones of Cantwell and Conlon}

Let $N$ be a compact, connected $n$-manifold and let $\omega \in \Omega^{1}(N)$ be a closed, nonsingular 1 -form. Then $\omega$ defines a codimension-1 foliation $\mathcal{F}$ of $N$. Thus we call $\omega$ a foliated form. Note that by Tischler's Theorem (see Candel and Conlon [3, Theorem 9.4.2]) $N$ admits such a form if and only if $N$ fibres over $S^{1}$. Now suppose that $\mathcal{F}$ is a depth-one foliation of a sutured manifold $(M, \gamma)$. Then there is a fibration $p: M_{0} \rightarrow S^{1}$. So the foliation determines a foliated class $[\omega] \in H^{1}(M ; \mathbb{R})$, where $\omega:=p^{*}(d t) \in \Omega^{1}\left(M_{0}\right)$ is the pullback of the standard form $d t \in \Omega^{1}\left(S^{1}\right)$ via the fibration $p$. Clearly $\omega$ defines a foliation $\mathcal{F}_{0}:=\mathcal{F} \mid M_{0}$. In particular, $\omega$ "blows up nicely" at $R(\gamma)$ (see Cantwell and Conlon [10, page 3.9]), which means that $\mathcal{F}_{0}$ can be completed to the foliation $\mathcal{F}$ by adjoining the connected components of $R(\gamma)$ as leaves. 
Let $\omega \in H^{1}(M ; \mathbb{R})$ and let $\mathcal{F}$ be a foliation determined by $\omega$. Cantwell and Conlon define a foliated ray $[\mathcal{F}]$ to be a ray $t \cdot \omega$ for $t \in \mathbb{R}, t>0$, in $H^{1}(M ; \mathbb{R})$ issuing from the origin [10, Corollary 4.4]. If $\mathcal{F}$ is a depth-zero or depth-one foliation, then the foliation ray is called a proper foliated ray $[10$, page 36$]$ and $[\mathcal{F}]$ is then given by $[\mathcal{F}]=\{t \cdot \lambda(\mathcal{F})\}_{t>0}$, since $\lambda(\mathcal{F})$ is precisely the foliated form determining $\mathcal{F}$.

In general, a foliated form defines foliations $\mathcal{F}$ which are tangent to $R(\gamma)$, have no holonomy, and their leaves are dense in $M_{0}$ (see Hector [16]). Moreover, any smooth foliation with holonomy only along $R(\gamma)$ is $C^{0}$ isotopic to a foliation defined by a foliated form [10, Corollary 4.4]. If $R(\gamma)=\varnothing$, then the foliated ray determines the foliation up to smooth isotopy (see Laudenbach and Blank [20] and Qué and Roussarie [24]). If a foliated form corresponds to an integral lattice point of $H^{1}(M ; \mathbb{R})$, then it determines a depth-zero or depth-one foliation, up to equivalence (see Theorem 3.8). Cantwell and Conlon [6] have shown that in all other cases the foliated ray also determines the foliation up to smooth-leaved isotopy.

Theorem 3.14 (Cantwell and Conlon [10, Theorem 1.1]) Let $(M, \gamma)$ be a sutured manifold. If there are depth-zero and depth-one foliations $\mathcal{F}$ of $(M, \gamma)$, then there are finitely many open, convex, polyhedral cones in $H^{1}(M)$, called foliation cones, having disjoint interiors and such that the foliated rays $[\mathcal{F}]$ are exactly those lying in one of these cones. The proper foliated rays are exactly the foliated rays through points of the integral lattice and determine the corresponding foliations up to isotopy.

Let $\mathfrak{C}(M, \gamma)$ denote the interior of the foliation cones in $H_{2}(M, \partial M ; \mathbb{R})$ obtained by Poincaré duality.

\section{Duality of the sutured Floer polytope and foliation cones}

As was remarked in Section 1, the moral of Lemma C is known to experts (personal correspondence with Conlon and Gabai), but the author was unable to find any written references to it. Cantwell and Conlon are preparing a paper [7] exploring the relationship of sutured manifold decomposition and foliations from the perspective of staircases and junctures that will include a proof of Lemma C. Nonetheless, for the reader's convenience, we give our own proof of Lemma $\mathrm{C}$, together with the minimal necessary background from foliation theory.

Therefore, the first part of this section is dedicated to the "classical" topology of taut depth-one foliations and the proof of Lemma $\mathrm{C}$ using Gabai's construction and the theory of spiral neighbourhoods given in Sections 3.2 and 3.3. The second part of this section covers the proofs of Theorems A and B. We conclude the paper by discussing some concrete examples that illustrate the duality of Theorem B. 


\subsection{The classical topology of depth-one foliations on sutured manifolds}

Let $(M, \gamma)$ be a sutured manifold as defined by Gabai.

Lemma C Suppose $(M, \gamma)$ is a connected sutured manifold. Let $(M, \gamma) \stackrel{S}{\wedge}\left(M^{\prime}, \gamma^{\prime}\right)$ be a surface decomposition along $S$ such that $\left(M^{\prime}, \gamma^{\prime}\right)$ is taut. Then $\left(M^{\prime}, \gamma^{\prime}\right)$ is a product sutured manifold if and only if either

(i) $R(\gamma)=\varnothing$ and $S$ is the fibre of a depth-zero foliation $\mathcal{F}$ given by a fibration $\pi: M \rightarrow S^{1}$, or

(ii) $R(\gamma) \neq \varnothing$ and $S$ can be spun along $R(\gamma)$ to be a leaf of a depth-one foliation $\mathcal{F}$ of $(M, \gamma)$.

Up to equivalence, all depth-zero and depth-one foliations of $(M, \gamma)$ are obtained from a surface decomposition resulting in a product sutured manifold.

Lemma 4.1 Let $(M, \gamma)$ be a connected sutured manifold such that $R(\gamma) \neq \varnothing$ and suppose $(M, \gamma) \stackrel{S}{\sim}\left(M^{\prime}, \gamma^{\prime}\right)$ is a well-groomed surface decomposition giving a product sutured manifold $\left(M^{\prime}, \gamma^{\prime}\right)$. Then there is a depth-one foliation on $(M, \gamma)$.

Proof This lemma is a particular case of Gabai [13, Theorem 5.1]; see Section 3.2.

We now remove the well-groomed condition, thereby proving the "only if' direction of Lemma C.

Lemma 4.2 Let $(M, \gamma)$ be a connected sutured manifold such that $R(\gamma) \neq \varnothing$ and suppose $(M, \gamma) \stackrel{S}{\rightsquigarrow}\left(M^{\prime}, \gamma^{\prime}\right)$ is a surface decomposition giving a product sutured manifold $\left(M^{\prime}, \gamma^{\prime}\right)$. Then there is a depth-one foliation on $(M, \gamma)$.

Proof We assume that the reader is familiar with the construction and the notation in Case 2 of Section 3.2. As before let $V$ be a component of $R_{+}(\gamma)$. Let $\mathcal{J}:=\left\{J_{1}, \ldots, J_{k}\right\}$ denote the connected components of $\partial S \cap V ; \mathcal{J}$ collects the junctures of $V$.

If $\left(M^{\prime}, \gamma^{\prime}\right)$ is connected, we apply the construction from Case 2 of Section 3.2 at every component of $R(\gamma)$ even though $S$ is not necessarily well-groomed. If $\left(M^{\prime}, \gamma^{\prime}\right)$ is not connected, we construct the foliation in the same way only starting from the decomposition along a surface $T$ in the same homology class as $S$, that is $[T]=$ $[S] \in H_{2}(M, \partial M)$, where $T$ has the following property: if $T^{\prime}$ is any maximal union of pairwise nonisotopic components of $T$, then $T^{\prime}$ does not disconnect $M$. See Turaev [29, Lemma 1.2] for a proof that such a $T$ exists. This gives a smooth foliation $\mathcal{F}$ 
of $(M, \gamma)$. To prove the lemma we need to show three things: that every leaf in the interior of $M$ is noncompact, that every such leaf is of depth-one (and hence totally proper), and that $\mathcal{F}$ is taut.

Firstly, if the spinning procedure were to yield a compact leaf in the interior that would mean that $\left[J_{i_{1}}\right]+\cdots+\left[J_{i_{n}}\right]=0$ for some subset $J_{i_{1}}, \ldots, J_{i_{n}}$ of junctures in $\mathcal{J}$. This is impossible if $\left(M^{\prime}, \gamma^{\prime}\right)$ is connected as cutting along $S$ would mean that $\left|R\left(\gamma^{\prime}\right)\right|>2$ contradicting that $\left(M^{\prime}, \gamma^{\prime}\right)$ is a product. Now, if $\left(M^{\prime}, \gamma^{\prime}\right)$ is not connected, note that since the homology class of each juncture is nondivisible, we may assume that $\left[J_{i_{k}}\right] \neq\left[J_{i_{l}}\right]$ for $k \neq l$; in other words, the junctures whose homology classes add up to zero come from $T^{\prime}$. Further, note that cutting along any component of $T \backslash T^{\prime}$ only splits off product manifolds. Together with the fact that $\left(M^{\prime}, \gamma^{\prime}\right)$ consists only of products, we therefore know that cutting along $T^{\prime}$ also gives some product manifold $(N, \delta)$. But $\left[J_{i_{1}}\right]+\cdots+\left[J_{i_{n}}\right]=0$ implies that cutting along $T^{\prime}$ gives $(N, \delta)$ with $|R(\delta)|>2$ contradicting that $(N, \delta)$ is a product manifold.

Secondly, let $L_{1}$ and $L_{2}$ be two arbitrary noncompact (and not necessarily distinct) leaves of $\mathcal{F}$. If we can show that $L_{1}$ is not asymptotic to $L_{2}$, then each noncompact leaf is totally proper and has depth-one. To show this we use the following observation. If $L_{1}$ is asymptotic to $L_{2}$, then for every point $x \in L_{2}$ and for each arc $a$ transverse to $\mathcal{F}$ and passing through $x$ the set $a \cap L_{1}$ clusters at $x$.

Let us label the noncompact leaves of $\mathcal{F}$ according to the level set of the product foliation $\mathcal{P}$ of $\left(M^{\prime}, \gamma^{\prime}\right)=(\Sigma \times[0,1], \partial \Sigma \times[0,1])$ that gives rise to each leaf. So for some $s, t \in(0,1)$, we have two (not necessarily distinct) leaves $L_{s}^{\prime}=\Sigma \times s$ and $L_{t}^{\prime}=\Sigma \times t$. Let $L_{s}$ and $L_{t}$ be the leaves of $\mathcal{F}$ such that $L_{s} \supset L_{s}^{\prime}$ and $L_{t} \supset L_{t}^{\prime}$.

Let $x$ be a point in $L_{t}$ and let $a$ be an arc in $M$ that is transverse to $\mathcal{F}$. We need to show that $a \cap L_{s}$ does not cluster around $x$. Suppose that $x$ is in $L_{t}^{\prime}$. Then $x$ is unaffected by the spinning of $\mathcal{F}$ near $R(\gamma)$. So we can choose a sufficiently small open neighbourhood $U_{x}$ of $x$ in $M$ such that $a \cap L_{s} \cap U_{x}=\varnothing$ if $s \neq t$, and $a \cap L_{s} \cap U_{x}=\{x\}$ if $s=t$. Therefore, $a \cap L_{s}$ does not cluster around $x$.

Now suppose that $x \notin L_{t}^{\prime}$. Then either $x \in D_{i}$ or $x \in K$. Recall that $D_{i}=J_{i} \times$ $[0,1] \times[0, \infty]$ and $K=(R(\gamma) \backslash N) \times[0, \infty]$, where $N$ was a neighbourhood of $J_{i}$ as described in the construction of $\mathcal{F}$. (For the construction of $\mathcal{F}$ see Section 3.2; note that there $D_{i}$ was parametrized differently as $J_{1} \times[-2,-1] \times[0, \infty]$.) In either case, $x$ is of the form $y \times(t+m)$, for some point $y$ in $J_{i} \times[0,1]$ or in $R(\gamma) \backslash N$ and some nonnegative integer $m$. Moreover, because of the choice of parametrisation and gluing given in (9), any point of $L_{s} \backslash L_{s}^{\prime}$ is of the form $z \times(s+n)$, for some nonnegative integer $n$. If $s \neq t$, then

$$
\min \{|s+m-t-n|: m, n \in \mathbb{Z}\}>0 .
$$


If $s=t$, then by definition of transversality the $\operatorname{arc} a$ can only intersect $L_{t}$ at points of the form $y \times(t+m)$, where the second coordinate points are discrete. In both cases there is again a small neighbourhood $U_{x}$ such that $a \cap L_{s} \cap U_{x}=\varnothing$, and $a \cap L_{s} \cap U_{x}=\{x\}$, respectively. So two noncompact leaves of $\mathcal{F}, L_{1}$ and $L_{2}$ are not asymptotic.

Lastly, $\mathcal{F}$ is taut for the same reason as when $S$ was well-groomed.

Before we proceed to prove the "if" direction of Lemma C, in Lemma 4.4, consider the following definition.

Definition 4.3 Let $\mathcal{F}$ be a depth-one foliation of $(M, \gamma)$. Suppose $L$ is a noncompact leaf of $\mathcal{F}$. Then a surface $\Sigma$ is obtained by truncating $L$, if $\Sigma=L \backslash \mathcal{N}$, for some spiral staircase neighbourhood $\mathcal{N}$ of $R(\gamma)$.

We can now summarise the content of Lemma 4.4: from a depth-one foliation $\mathcal{F}$ of $(M, \gamma)$, one can obtain a surface $S$ giving a product decomposition by truncating an arbitrary noncompact leaf $L$ of $\mathcal{F}$. Morally, we take a leaf and remove its ends and what we are left with is the surface. The details follow.

Lemma 4.4 Suppose that $\mathcal{F}$ is a depth-one foliation on $(M, \gamma)$. Then there exists a surface decomposition $(M, \gamma) \stackrel{S}{\rightsquigarrow}\left(M^{\prime}, \gamma^{\prime}\right)$ giving a product manifold.

Proof Lemma 3.5 says that a depth-one foliation $\mathcal{F}$ determines a fibration $M_{0} \rightarrow S^{1}$. For each component $V$ of $R(\gamma)$, let $\mathcal{N}_{V}$ be a spiral staircase neighbourhood of $V$ associated to $\mathcal{F}$ and to some transverse foliation $\mathcal{L}$ (see Section 3.3 for details and notation). Recall that the construction of $\mathcal{N}_{V}$ starts by choosing a leaf $L_{0}$ and an end $e$ of $L_{0}$ converging to $V$, followed by a choice of a fundamental neighbourhood system $\mathcal{U}$ of $e$, which yields a set of junctures $J_{1}, J_{2}, \ldots, J_{k}$. The boundary of each $\mathcal{N}_{V}$ is $V \cup B_{i} \cup T_{i}$, for some $i \in \mathbb{N}$, where $B_{i} \subset L_{0}$, and $T_{i}$ is obtained by flowing $J_{i}$ along $\mathcal{L}$.

Set $\mathcal{N}:=\bigcup_{V \subset R(\gamma)} \mathcal{N}_{V}$. Then $\bar{M}:=M \backslash \mathcal{N}$ is clearly homeomorphic to $M$ and determines a sutured manifold $(\bar{M}, \bar{\gamma})$ with the same sutures as $M$. Consider the effect of removing $\mathcal{N}$ on the foliation $\mathcal{F}$. Let $L$ and $L^{\prime}$ be two leaves of $\mathcal{F}$ transverse to $\mathcal{N}$. This implies that $\bar{L}:=\bar{M} \cap L$ is homeomorphic to $\bar{L}^{\prime}:=\bar{M} \cap L^{\prime}$ via a map defined using $\mathcal{L}$.

The boundary $\partial \bar{M}$ is the union of two subsets $T$ and $B$, where $T$ is the subset of $\partial \mathcal{N}$ such that the leaves of $\mathcal{F} \mid \bar{M}$ are transverse to $T$, and $B:=\partial \bar{M} \backslash \operatorname{Int} T$. Note that $B \subset \bigcup_{i=1}^{k} \bar{L}_{i}$, where $L_{i}$ are some leaves of $\mathcal{F}$ and $\bar{L}_{i}:=L_{i} \cap \bar{M}$. Set $K:=\bigcup_{i=1}^{k} \bar{L}_{i}$. 
As $\bar{L}$ is homeomorphic to $\bar{L}^{\prime}$, the manifold $M \backslash K$ is a fibration over the unit interval with fibre $\bar{L}$.

Set $S:=K \backslash B$. Then $S$ is a decomposing surface of $(\bar{M}, \bar{\gamma})$, so we have a decomposition $(\bar{M}, \bar{\gamma}) \stackrel{S}{\rightarrow}\left(M^{\prime}, \gamma^{\prime}\right)$ and we know that $M^{\prime} \cong \bar{L} \times I$. It remains to show that $\gamma^{\prime}=\partial \bar{L} \times I$. Choose the orientation of $S$ to be opposite from the orientation of $K$. By the definition of a surface decomposition

$$
\gamma^{\prime}:=\left(\gamma \cap M^{\prime}\right) \cup\left(S_{+} \cap R_{-}(\bar{\gamma})\right) \cup\left(S_{-} \cup R_{+}(\bar{\gamma})\right) .
$$

Further, by the definition of $\mathcal{F}$ all of the fibres are transverse to $\gamma$. A careful consideration of orientations now shows that $\left(S_{+} \cap R_{-}(\bar{\gamma})\right) \cup\left(S_{-} \cup R_{+}(\bar{\gamma})\right)=B$, possibly after a small isotopy of the boundary. Thus, $\gamma^{\prime}$ is precisely the subset of $\partial M^{\prime}$ to which the fibres of the fibration are transverse; in other words, $\gamma^{\prime}=\partial L^{\prime} \times I$.

It follows that $(M, \gamma) \stackrel{S}{\rightsquigarrow}\left(M^{\prime}, \gamma^{\prime}\right)$ yields a product sutured manifold $\left(M^{\prime}, \gamma^{\prime}\right)$.

Remark 4.5 Suppose that we are given a taut depth-one foliation as in the statement of Lemma 4.4. Note that if $\alpha$ is a loop in $M$, then $\langle\lambda(\mathcal{F}),[\alpha]\rangle$ is the signed intersection number of $\alpha$ with a noncompact leaf $L$. Truncating $L$ by a sufficiently small spiral staircase neighbourhood in Lemma 4.4, we have that $\langle P D \circ[S],[\alpha]\rangle=\langle\lambda(\mathcal{F}),[\alpha]\rangle$ for any loop $\alpha$. Hence $\lambda(\mathcal{F})=P D \circ[S]$, where $S$ is the decomposing surface obtained by truncating $L$.

Conversely, suppose that we are given a decomposition $(M, \gamma) \stackrel{S}{\rightsquigarrow}\left(M^{\prime}, \gamma^{\prime}\right)$ yielding a product manifold as in Lemma 4.2. Note that if the homology class $[S]$ is divisible, that is $[S]=n \beta$ for some $n \in \mathbb{N}$ and $\beta$ a nondivisible class, then we can find a surface $T$ comprising $n$ parallel copies of $T^{\prime}$, for some surface $T^{\prime}$ satisfying the property described in the proof of Lemma 4.2 and with $\left[T^{\prime}\right]=\beta$. Working through Gabai's construction we see that the foliation created by spinning $T^{\prime}$ along $R(\gamma)$ is isotopic to the one created by spinning $T$ along $R(\gamma)$. Morally speaking, spinning $T^{\prime}$ along $R(\gamma)$ infinitely many times, is the same as spinning $n$ parallel copies of $T^{\prime}$ along $R(\gamma)$ infinitely many times. A consequence of this is that if we are given a foliation $\mathcal{F}$ created by spinning along $T$, truncating a noncompact leaf $L$ returns the decomposing surface as $T^{\prime}$. This is not a surprise, as from Remark 3.6 we know that $\lambda(\mathcal{F})$, which is in this case equal to $\beta$, is a nondivisible homology class.

The following is a corollary of Remark 4.5 and of Theorem 3.8.

Corollary 4.6 Let $\mathcal{F}$ and $\mathcal{F}^{\prime}$ be two depth-one foliations of $(M, \gamma)$, together with the decomposing surfaces from Lemma 4.4, $S$ and $S^{\prime}$, respectively. Then $\mathcal{F}$ is equivalent to $\mathcal{F}^{\prime}$ if and only if $[S]=\left[S^{\prime}\right] \in H_{2}(M, \partial M)$. 
Proof of Lemma C When $R(\gamma)=\varnothing$ and $\left(M^{\prime}, \gamma^{\prime}\right)$ is a product, then $\mathcal{F}$ is a fibration. Conversely, when $\mathcal{F}$ is a fibration, cutting along a fibre gives a product. For the depth-one case Lemmas 4.2 and 4.4 each prove one direction of the theorem.

Remark 4.7 Recall that in Remark 3.4 we distinguished two cases when $M$ fibres over $S^{1}$. The first case is when $R(\gamma)=\varnothing$ and the fibration is a depth-zero foliation (think of $S^{1} \times D^{2}$ fibred with $T(\gamma)=S^{1} \times \partial D^{2}$ ). The second case is when $A(\gamma) \neq \varnothing$, and the fibres are not transverse to $\gamma$ or not tangential to $R(\gamma)$ (think $S^{1} \times D^{2}$ with two parallel sutures). In both cases cutting along a fibre can result in a connected product sutured manifold. From the proof of Lemma $\mathrm{C}$, it is now evident that in the first case our construction from the surface decomposition recovers the depth-zero foliation (that is, the fibration), but that in the second case we construct a depth-one foliation.

Note that in Lemma $\mathrm{C}$, we do not restrict to balanced sutured manifolds, therefore $R(\gamma)=\varnothing$ can happen. However, in order to work with the sutured Floer polytope in the following section, we must restrict to strongly balanced sutured manifolds, so only the latter case of fibring can occur.

\subsection{The duality}

Let $(M, \gamma)$ be a strongly balanced sutured manifold. Recall that given a decomposing surface $S$, there exists a set of outer $\operatorname{Spin}^{c}$ structures denoted by $O_{S}$; see Definition 2.11 .

Definition 4.8 Let $(M, \gamma)$ be a taut, strongly balanced sutured manifold.

(i) A $\operatorname{Spin}^{c}$ structure $\mathfrak{s}$ is called extremal if there exists a surface $S$ such that $\{\mathfrak{s}\}=O_{S} \cap S(M, \gamma)$. Then $\mathfrak{s}$ is extremal with respect to $\alpha:=[S]$, and this is equivalent to saying that $\alpha(\mathfrak{s})>\alpha(\mathfrak{t})$ for any other $\mathfrak{t} \in S(M, \gamma)$ (see Theorems 2.12 and 2.8).

(ii) The polytope $P(M, \gamma)$ is said to have an extremal $\mathbb{Z}$ at $\mathfrak{s}$, if $\mathfrak{s}$ is extremal and $\operatorname{SFH}(M, \gamma, \mathfrak{s})=\mathbb{Z}$.

The following lemma is a direct consequence of Juhász's work; it is useful for us to write it in the terminology from Definition 4.8.

Lemma 4.9 Let $(M, \gamma)$ be a taut, strongly balanced sutured manifold with $H_{2}(M)=0$. Then $\mathfrak{s}$ is an extremal $\mathbb{Z}$ with respect to a homology class $\alpha$ if and only if there exists a surface decomposition $(M, \gamma) \stackrel{S}{\sim}\left(M^{\prime}, \gamma^{\prime}\right)$ such that $\left(M^{\prime}, \gamma^{\prime}\right)$ is a product and that $\alpha=[S]$. 
Proof Let $\mathfrak{s}$ carry a $\mathbb{Z}$ extremal to $\alpha$. Then by Theorem 2.8 , there exists a surface decomposition $(M, \gamma) \stackrel{S}{\rightsquigarrow}\left(M^{\prime}, \gamma^{\prime}\right)$ such that $\left(M^{\prime}, \gamma^{\prime}\right)$ is taut, $\alpha=[S]$ and $S F H\left(M^{\prime}, \gamma^{\prime}\right)=$ $\operatorname{SFH}(M, \gamma, \mathfrak{s})=\mathbb{Z}$. Theorem 2.6 implies that $\left(M^{\prime}, \gamma^{\prime}\right)$ is a product. Conversely, if such a decomposition is given, then

$$
\bigoplus_{\mathfrak{s} \in O_{S}} \operatorname{SFH}(M, \gamma, \mathfrak{s})=\operatorname{SFH}_{\alpha}(M, \gamma)=\operatorname{SFH}\left(M^{\prime}, \gamma^{\prime}\right)=\mathbb{Z}
$$

where the second equality comes from Theorem 2.12 and the third from Theorem 2.6. The result follows.

Remark 4.10 Note that in Lemma 4.9 if $\mathfrak{s}$ is extremal with respect to some $\alpha$, then $\alpha$ can be assumed to be nondivisible (otherwise $\alpha=n \beta$ for some nondivisible $\beta$ and some $n \in \mathbb{N}$, and $\mathfrak{s}$ is then extremal with respect to the nondivisible class $\beta$ ). Using the same lemma of Turaev [29, Lemma 1.2] as in Remark 4.5 implies that Lemma 4.9 holds when $\alpha$ is taken to be nondivisible.

Lemmas $\mathrm{C}$ and 4.9 lead to Theorem A.

Theorem A Suppose $(M, \gamma)$ is a taut, strongly balanced sutured manifold with $H_{2}(M ; \mathbb{Z})=0$, and let $P(M, \gamma)$ denote its sutured polytope. Then $P(M, \gamma)$ has an extremal $\mathbb{Z}$ at a Spin ${ }^{c}$ structure $\mathfrak{s}$ if and only if there exists a taut depth-one foliation $\mathcal{F}$ of $(M, \gamma)$ whose sole compact leaves are the connected components of $R(\gamma)$ and such that $\mathfrak{s}$ is extremal with respect to $P D \circ \lambda(\mathcal{F})$.

Proof Let $\mathfrak{s}$ be an extremal $\mathbb{Z}$ of $P(M, \gamma)$. By Lemma 4.9, there exists a decomposing surface $S$ with $(M, \gamma) \stackrel{S}{\rightsquigarrow}\left(M^{\prime}, \gamma^{\prime}\right)$ such that $[S]=\alpha$ and $\left(M^{\prime}, \gamma^{\prime}\right)$ is a product. By Remark 4.10 we may assume $\alpha$ is nondivisible. By Lemma $C$, we can construct $\mathcal{F}$, and by Remark 4.5 we have that $\lambda(\mathcal{F})=P D \circ[S]$. Conversely, given such a foliation $\mathcal{F}$, by the proof of Lemma $\mathrm{C}$, truncating a noncompact leaf $L$ gives a decomposing surface $S$ with $\lambda(\mathcal{F})=P D \circ[S]$. Since we get a product $\left(M^{\prime}, \gamma^{\prime}\right)$ when decomposing along $S$, applying Lemma 4.9 completes the proof.

The disadvantage of the sutured Floer polytope is that it is well defined only up to translation in $H^{2}(M, \partial M ; \mathbb{R})$. Thus, a choice needs to be made for there to exist a well-defined dual polytope. However, without making any choices we can define the dual sutured cones $Q(M, \gamma)$ in $H_{2}(M, \partial M ; \mathbb{R})$.

Let $P$ be a polytope given, up to translation, by vertices $v_{1}, \ldots, v_{n}$ living in a vector space $V$ over some field $\mathbb{F}$. Then, as we said in the Section 1 , the dual cones $Q$ 
can be defined to be a collection of polyhedral cones $Q_{1}, \ldots, Q_{n}$ in the dual space $V^{*}=\operatorname{Hom}(V, \mathbb{F})$, where

$$
\left.Q_{i}:=\left\{v^{*} \in V^{*}: v^{*}\left(v_{i}-v_{j}\right)>0\right) \text { for } i \neq j\right\} .
$$

Definition 4.11 Define the dual sutured cones $Q(M, \gamma)$ in $H_{2}(M, \partial M ; \mathbb{R})$ to be the dual cones of the sutured polytope $P(M, \gamma)$, with each labeled by the corresponding extremal $\operatorname{Spin}^{c}$ of $P(M, \gamma)$. The cones that correspond to extremal $\mathbb{Z}$ vertices of $P(M, \gamma)$ are called the extremal $\mathbb{Z}$ cones and are denoted by $Q_{\mathbb{Z}}(M, \gamma)$.

In the introduction we mentioned how one defines a dual cone of any polytope in an affine space given just by its vertices. Definition 4.11 just repeats this definition only in the language that is most useful here.

Lemma 4.12 Let $(M, \gamma)$ be a taut, strongly balanced sutured manifold with $H_{2}(M)=$ 0 . Then the closure of each subset $C_{\mathfrak{s}}$ of the dual sutured cones $Q(M, \gamma)$ is indeed a convex, polyhedral cone. Also, if $\operatorname{dim} P(M, \gamma)=b_{1}(M)$, then the closure of $Q(M, \gamma)$ covers all of $\mathrm{H}_{2}(M, \partial M ; \mathbb{R})$.

Proof This is just stating that $Q(M, \gamma)$ is defined as a dual to $P(M, \gamma)$.

Theorem B Let $(M, \gamma)$ be a taut, strongly balanced sutured manifold with $H_{2}(M)=0$. The extremal $\mathbb{Z}$ cones of $Q(M, \gamma)$ are precisely the foliation cones $\mathfrak{C}(M, \gamma)$ defined by Cantwell and Conlon in [10] (see Theorem 3.14).

Proof By Theorem 3.14 the foliation cones $\mathfrak{C}(M, \gamma)$ are open, convex, polyhedral cones in $H_{2}(M, \partial M ; \mathbb{R})$. Further, the nondivisible integral homology classes $\alpha \in H_{2}(M, \partial M)$ in $\mathfrak{C}(M, \gamma)$ are precisely those for which there exists a foliation $\mathcal{F}$ of depth-one such that $\lambda(\mathcal{F})=P D \circ \alpha$. By Theorem A and Remark 4.5, it follows extremal $\mathbb{Z}$ points of $P(M, \gamma)$ correspond precisely to such foliations. In particular, $Q_{\mathbb{Z}}$ is a collection of open, convex, polyhedral cones whose nondivisible integral homology classes correspond to depth-one foliations via the same correspondence of $\alpha=P D \circ \lambda(\mathcal{F})$. Now, if $C_{1}$ and $C_{2}$ are two unions of open, convex, polyhedral cones that are defined by integral inequalities and that coincide on integral points, that is $C_{1} \cap \mathbb{Z}^{n}=C_{2} \cap \mathbb{Z}^{n}$, then $C_{1}$ and $C_{2}$ coincide on all points. As both $\mathfrak{C}(M, \gamma)$ and $Q_{\mathbb{Z}}(M, \gamma)$ are defined by inequalities with integral coefficients (for the statement about $\mathfrak{C}(M, \gamma)$, see Candel and Conlon [3, Corollary 5.20]), it follows that $\mathfrak{C}(M, \gamma)$ is the same as $Q_{\mathbb{Z}}(M, \gamma)$. 
Remark 4.13 The requirement that $H_{2}(M)=0$ comes from the fact that sutured Floer homology of a manifold is defined only if each component of the boundary contains a suture. Therefore, cutting along a closed surface produces a manifold for which the sutured Floer homology is undefined. It is clear that if there is a decomposition $(M, \gamma) \stackrel{S}{\sim}\left(M^{\prime}, \gamma^{\prime}\right)$ such that $\left(M^{\prime}, \gamma^{\prime}\right)$ is a product, then $S$ cannot have closed components. This means that if we know there is such a decomposition, we can identify an extremal $\mathbb{Z}$, that is a vertex of the polytope that carries $\mathbb{Z}$ and that corresponds to the product manifold obtained by cutting along $S$, without the assumption that $H_{2}(M)=0$. However, conversely, if there is an extremal $\mathbb{Z}$, there exists a surface decomposition giving a product according to Juhász's decomposition formulas [18, Theorem 1.3] and [19, Corollary 4.15], if we know that the relevant homology classes have an open surface as their representative. Obviously such representatives are guaranteed to exist when $H_{2}(M)=0$.

The assumption $H_{2}(M)=0$ seems a bit too strong, especially since we are only interested in $\operatorname{Spin}^{c}$ structures $\mathfrak{s}$, such that $P(M, \gamma)$ has an extremal $\mathbb{Z}$ at $\mathfrak{s}$. One might hope that in this special case it can be shown that all homology classes of surfaces giving extremal $\mathbb{Z}$ indeed do have open surfaces as representatives.

We conclude with a corollary that describes the sutured manifold analogue of the Thurston norm and its fibred faces for closed 3-manifolds.

Denote by $B_{y}$ the polytope in $H_{2}(M, \partial M ; \mathbb{R})$ that is the unit ball of the Juhász's seminorm $y$ described in Remark 2.17. The faces of $B_{y}$ that are dual to extremal $\mathbb{Z}$ Spin $^{c}$ structures in $-P(M, \gamma)$ are called the foliated faces.

Corollary 4.14 If $(M, \gamma)$ is a taut, strongly balanced sutured manifold with $H_{2}(M)=$ 0 , then each foliation cone of $\mathfrak{C}(M, \gamma)$ is subtended by a foliated face of $B_{y}$.

As $y$ involves the somewhat artificial choice of putting the centre of mass of $P(M, \gamma)$ at $0 \in H^{2}(M, \partial M ; \mathbb{R})$, the obvious question is why is this corollary not phrased in terms of $y_{t}$. Of course, a similar statement could be made for the unit ball of the geometric sutured function $y_{t}$, however only if it makes sense to talk about the unit ball, that is, if $y_{t}$ is at least a seminorm.

\subsection{Examples}

Finally, we illustrate Theorem B by checking that the examples of foliations cones computed by Cantwell and Conlon $[8 ; 10]$ are indeed dual to the associated sutured Floer polytopes. 
Let $L$ be a knot or link in $S^{3}$, and $R$ a minimal genus Seifert surface of $L$. Then denote by $S^{3}(R)$ the strongly balanced sutured manifold obtained by removing an open neighbourhood of $R$ from $S^{3}$, that is, $S^{3}(R):=\left(S^{3} \backslash \operatorname{Int}(R \times I), \partial R \times I\right)$.

Let $P(2 r, 2 s, 2 t)$ denote the standard three-component pretzel link, and let $R$ be the Seifert surface obtained by the Seifert algorithm. Examples 2 and 5 from [10] describe the foliation cones of $S^{3}(R)$ for $P(2,2,2)$ and $P(2,4,2)$, respectively. The sutured Floer polytopes for these examples were computed by Friedl, Juhász and Rasmussen [12, Example 8.6], and it is not hard to see that they are indeed dual to the foliation cones.

Example 4 in [10] describes the foliation cones of $S^{3}(R)$ for a 2-component link and the Seifert surface given in Figure 6.

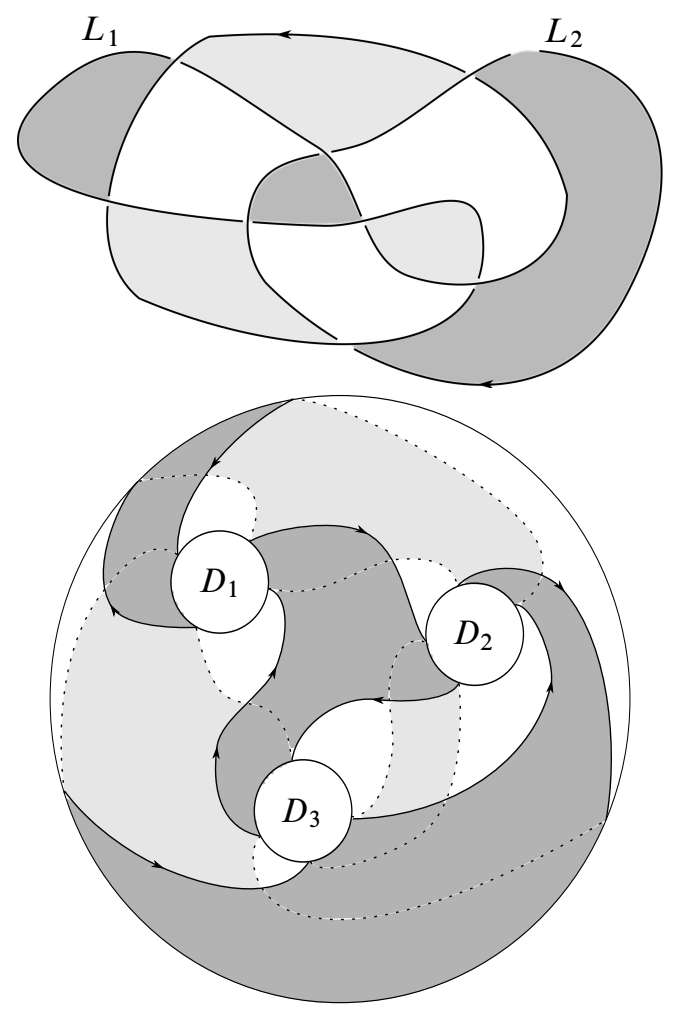

Figure 6: The 2-component link and its Seifert surface

As this is a nonsplit alternating link, by [12, Corollary 6.11] it follows that $S^{3}(R)$ is a sutured $L$-space, that is, the group $\operatorname{SFH}(M, \gamma, \mathfrak{s})$ is either trivial or isomorphic to $\mathbb{Z}$ for every $\operatorname{Spin}^{c}$ structure $\mathfrak{s}[12$, Corollary 6.6]. Therefore, $\operatorname{SFH}(M, \gamma)$ and, in 
particular $P(M, \gamma)$, can easily be computed from the map $\pi_{1}\left(R_{-}(\gamma)\right) \rightarrow \pi_{1}(M)$ using Fox calculus [12, Proposition 1.2].

Convention 4.15 For the remainder of the paper, homology groups are understood to be taken with coefficients in $\mathbb{R}$.

First, let us describe the foliation cones. Considering the isomorphism given by Poincaré duality $H^{1}(M)=H_{2}(M, \partial M)$, we take the foliation cones to live in $H_{2}(M, \partial M)=\mathbb{R}^{3}$. Following Cantwell and Conlon [10, Example 4, Figure 14], denote by $e_{1}, e_{2}, e_{3}$ the basis of $H_{2}(M, \partial M)$, given by the disks $D_{i}$ in Figure 6 . Let $e_{0}:=-\left(e_{1}+e_{2}+e_{3}\right)$. Then there are five convex foliation cones whose closures cover all of $H_{2}(M, \partial M)$ : four of the cones are 3-sided, and the fifth is 4-sided. The cones are determined by rays through five points

$$
-e_{2}-e_{3}, \quad e_{2},-e_{0}, \quad e_{3},-e_{1} \text {. }
$$

In Figure 7 these points have been connected in such a way that the sides of the pyramid subtend each cone. This is the easiest way for visualising the duality with the sutured Floer polytope.

Let $f_{1}, f_{2}, f_{3}$ be the basis of $H^{2}(M, \partial M)$ dual to $e_{1}, e_{2}, e_{3}$; here "dual" refers to the duality of $H^{2}(M, \partial M)$ and $H_{2}(M, \partial M)$ as vector spaces. Using the same notation, and the method of computation via Fox calculus, we find that the sutured Floer polytope $P\left(S^{3}(R)\right)$ in $H^{2}(M, \partial M)$ is a pyramid with a rectangular basis given by the vectors (up to translation)

$$
f_{2}+f_{3}, \quad f_{2}, \quad f_{3}, \quad f_{1}+f_{3}, \quad f_{1}+f_{2},
$$

where $f_{2}+f_{3}$ is the apex of the pyramid in Figure 7 .

To compute the dual of $P\left(S^{3}(R)\right)$, we first have to find the centre of mass $p$ of the polytope, then translate the polytope so that the centre of mass is at $0 \in H^{2}(M, \partial M)$. A bit of elementary geometry shows that $p=\frac{1}{5}\left(2 f_{1}+3 f_{2}+3 f_{3}\right)$ in the current coordinate system. Translate the polytope, or equivalently change the coordinates, so that $p=0$. The dual cones of $P\left(S^{3}(R)\right)$ are then given by five rays normal to the five sides of $P\left(S^{3}(R)\right)$. Using symmetries of the polytope it is not hard to compute that these rays precisely pass through $-e_{2}-e_{3}, e_{2},-e_{0}, e_{3},-e_{1}$, which described the foliation cones.

Remark 4.16 In all of the above examples, $x^{s}=z$. That is, the sutured Thurston norm of $(M, \partial M)$ agrees with the symmetrised sutured seminorm $z(\alpha)=\frac{1}{2}(y(\alpha)+y(-\alpha))$. This equality does not hold in general, as was shown by Friedl, Juhász and Rasmussen [12, Proposition 7.16] using an example of Cantwell and Conlon [8, Example 2]. 


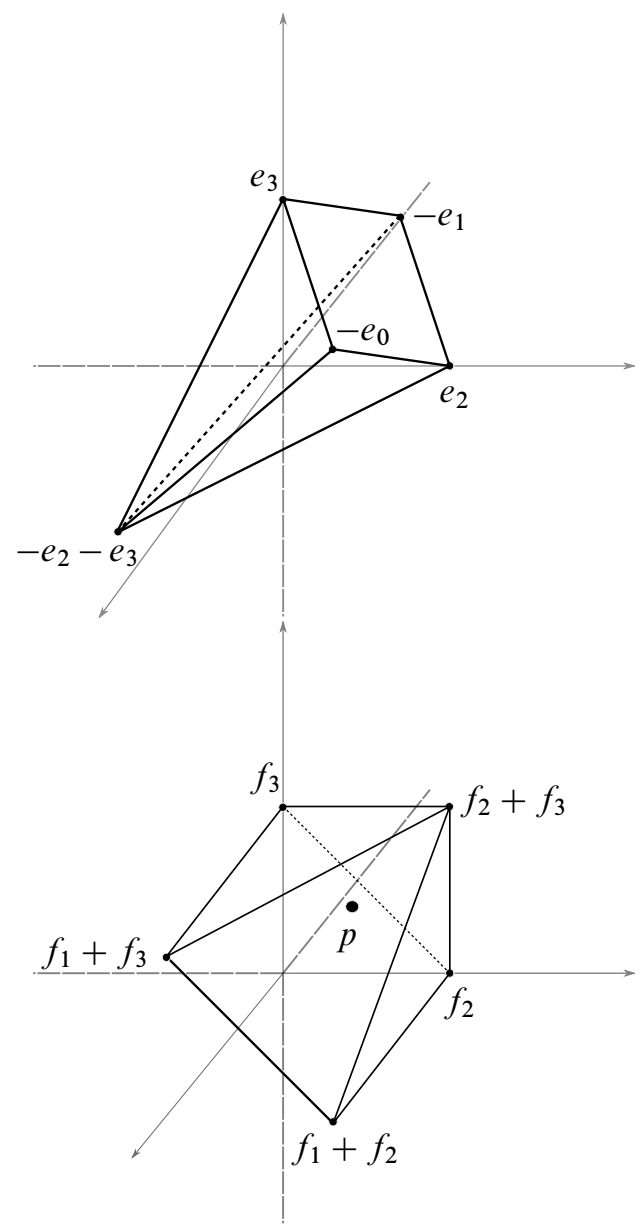

Figure 7: Left: the foliation cones given by rays through the vertices of the pyramid in $H_{2}(M, \partial M)=\mathbb{R}^{3}$; right: the sutured Floer polytope given up to translation in $H^{2}(M, \partial M)=\mathbb{R}^{3}$, with $p$ the centre of mass.

From their respective computations it is not hard to check that the sutured Floer polytope and the foliation cones are dual.

Remark 4.17 In [1] we show that there exists an infinite family of knots and pairs of Seifert surfaces $R_{1}$ and $R_{2}$ associated to each knot, where the polytopes $P\left(S^{3}\left(R_{1}\right)\right)$ and $P\left(S^{3}\left(R_{2}\right)\right)$ are not affine isomorphic. In other words, the sutured Floer polytope of $S^{3}(R)$ is not a knot invariant, and as the polytopes are defined by vertices carrying extremal $\mathbb{Z}$, it follows by Theorem $B$ that the foliation cones of a Seifert surface complement are also not a knot invariant. 


\section{References}

[1] I Altman, Sutured Floer homology distinguishes between Seifert surfaces, Topology Appl. 159 (2012) 3143-3155 MR2948273

[2] D Calegari, Foliations and the geometry of 3-manifolds, Oxford Math. Monographs, Oxford Uni. Press (2007) MR2327361

[3] A Candel, L Conlon, Foliations I, Graduate Studies in Mathematics 23, Amer. Math. Soc. (2000) MR1732868

[4] A Candel, L Conlon, Foliations II, Graduate Studies in Mathematics 60, Amer. Math. Soc. (2003) MR1994394

[5] J Cantwell, L Conlon, Handel-Miller theory and finite depth foliations arXiv: 1006.4525

[6] J Cantwell, L Conlon, Open saturated sets without holonomy, preprint

[7] J Cantwell, L Conlon, Smoothability of Gabai's foliations, in preparation

[8] J Cantwell, L Conlon, The sutured Thurston norm arXiv:math/060653

[9] J Cantwell, L Conlon, Isotopy of depth one foliations, from: "Geometric study of foliations", World Sci. Publ. (1994) 153-173 MR1363723

[10] J Cantwell, L Conlon, Foliation cones, from: "Proceedings of the Kirbyfest", Geom. Topol. Monogr. 2 (1999) 35-86 MR1734401

[11] S R Fenley, End periodic surface homeomorphisms and 3-manifolds, Math. Z. 224 (1997) 1-24 MR1427700

[12] S Friedl, A Juhász, J Rasmussen, The decategorification of sutured Floer homology, J. Topol. 4 (2011) 431-478 MR2805998

[13] D Gabai, Foliations and the topology of 3-manifolds, J. Differential Geom. 18 (1983) 445-503 MR723813

[14] D Gabai, Foliations and the topology of 3-manifolds II, J. Differential Geom. 26 (1987) 461-478 MR910017

[15] P Ghiggini, Knot Floer homology detects genus-one fibred knots, Amer. J. Math. 130 (2008) 1151-1169 MR2450204

[16] G Hector, Croissance des feuilletages presque sans holonomie, from: "Differential topology, foliations and Gelfand-Fuks cohomology", Lecture Notes in Math. 652, Springer, Berlin (1978) 141-182 MR505659

[17] A Juhász, Holomorphic discs and sutured manifolds, Algebr. Geom. Topol. 6 (2006) 1429-1457 MR2253454

[18] A Juhász, Floer homology and surface decompositions, Geom. Topol. 12 (2008) 299350 MR2390347 
[19] A Juhász, The sutured Floer homology polytope, Geom. Topol. 14 (2010) 1303-1354 MR2653728

[20] F Laudenbach, S Blank, Isotopie de formes fermées en dimension trois, Invent. Math. 54 (1979) 103-177 MR550181

[21] Y Ni, Heegaard Floer homology and fibred 3-manifolds, Amer. J. Math. 131 (2009) 1047-1063 MR2543922

[22] P Ozsváth, Z Szabó, Holomorphic disks and genus bounds, Geom. Topol. 8 (2004) 311-334 MR2023281

[23] P Ozsváth, Z Szabó, Holomorphic disks and topological invariants for closed threemanifolds, Ann. of Math. 159 (2004) 1027-1158 MR2113019

[24] N V Quê, R Roussarie, Sur l'isotopie des formes fermées en dimension 3, Invent. Math. 64 (1981) 69-87 MR621770

[25] M Scharlemann, Sutured manifolds and generalized Thurston norms, J. Differential Geom. 29 (1989) 557-614 MR992331

[26] S Schwartzman, Asymptotic cycles, Ann. of Math. 66 (1957) 270-284 MR0088720

[27] D Sullivan, Cycles for the dynamical study of foliated manifolds and complex manifolds, Invent. Math. 36 (1976) 225-255 MR0433464

[28] W P Thurston, A norm for the homology of 3-manifolds, Mem. Amer. Math. Soc. 339, Amer. Math. Soc. (1986) MR823443

[29] V G Turaev, A homological estimate for the Thurston norm arXiv:math/0207267

[30] V G Turaev, Euler structures, nonsingular vector fields, and Reidemeister-type torsions, Izv. Akad. Nauk SSSR Ser. Mat. 53 (1989) 607-643, 672 MR1013714

Mathematics Institute, University of Warwick

Coventry CV4 7AL, UK

irida.altman@gmail.com

Received: 9 May 2013 Revised: 21 November 2013 
